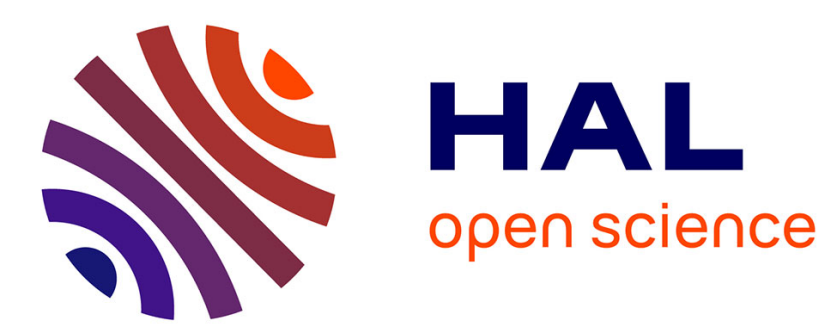

\title{
Energy minimization problem in two-level dissipative quantum control: meridian case
}

Bernard Bonnard, Olivier Cots, Nataliya Shcherbakova

\section{To cite this version:}

Bernard Bonnard, Olivier Cots, Nataliya Shcherbakova. Energy minimization problem in two-level dissipative quantum control: meridian case. Journal of Mathematical Sciences, 2013, vol. $195\left(\mathrm{n}^{\circ} 3\right)$, pp. 311-335. 10.1007/s10958-013-1582-4 . hal-01122070

\section{HAL Id: hal-01122070 \\ https://hal.science/hal-01122070}

Submitted on 3 Mar 2015

HAL is a multi-disciplinary open access archive for the deposit and dissemination of scientific research documents, whether they are published or not. The documents may come from teaching and research institutions in France or abroad, or from public or private research centers.
L'archive ouverte pluridisciplinaire HAL, est destinée au dépôt et à la diffusion de documents scientifiques de niveau recherche, publiés ou non, émanant des établissements d'enseignement et de recherche français ou étrangers, des laboratoires publics ou privés. 


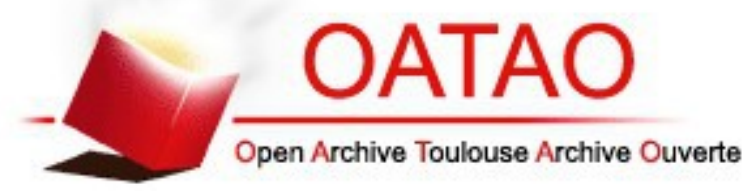

\section{Open Archive TOULOUSE Archive Ouverte (OATAO)}

OATAO is an open access repository that collects the work of Toulouse researchers and makes it freely available over the web where possible.

This is an author-deposited version published in : http://oatao.univ-toulouse.fr/ Eprints ID : 12315

To link to this article : DOI :10.1007/s10958-013-1582-4

URL : http://dx.doi.org/10.1007/s10958-013-1582-4

To cite this version : Bonnard, Bernard and Cots, Olivier and Shcherbakova, Nataliya Energy minimization problem in two-level dissipative quantum control: meridian case. (2013) Journal of Mathematical Sciences, vol. $195\left(\mathrm{n}^{\circ}\right.$ 3). pp. 311-335. ISSN 1072-3374

Any correspondance concerning this service should be sent to the repository administrator: staff-oatao@,listes-diff.inp-toulouse.fr 


\title{
ENERGY MINIMIZATION PROBLEM \\ IN TWO-LEVEL DISSIPATIVE QUANTUM CONTROL: MERIDIAN CASE
}

\author{
B. Bonnard, O. Cots, and N. Shcherbakova
}

\begin{abstract}
We analyze the energy-minimizing problem for a two-level dissipative quantum system described by the Kossakowsky-Lindblad equation. According to the Pontryagin maximum principle (PMP), minimizers can be selected among normal and abnormal extremals whose dynamics are classified according to the values of the dissipation parameters. Our aim is to improve our previous analysis from [5] concerning 2D solutions in the case where the Hamiltonian dynamics are integrable.
\end{abstract}

\section{CONTENTS}

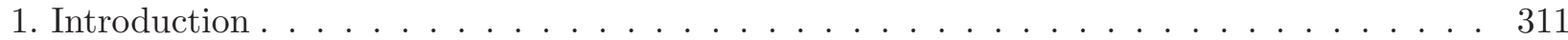

2. Abnormal Extremals in the Energy Minimization Problem . . . . . . . . . . . . . 313

3. Normal Case . . . . . . . . . . . . . . . . . . . . . . . . . . . . . . . 314

4. Integrable Case . . . . . . . . . . . . . . . . . . . . . . 316

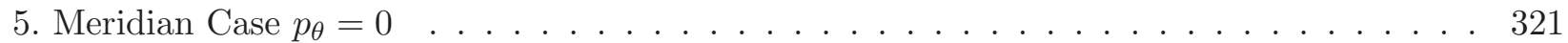

6. Numerical Computation of Cut and Conjugate Loci _ . . . . . . . . . . . . 326

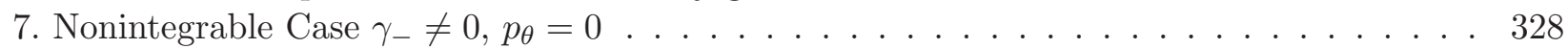

8. Summary of the Results and Possible Extensions . . . . . . . . . . . . . . . 331

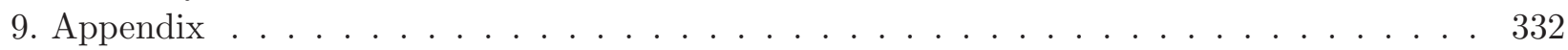

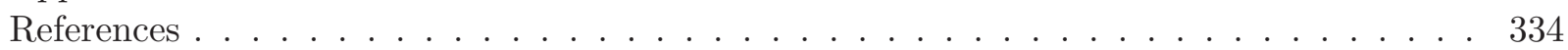

\section{Introduction}

Quantum control is one of the most challenging applications of modern control theory. Indeed, the problem of inducing transitions between molecular levels or spin states is relevant to many applications of chemical physics: from nuclear magnetic resonance, to spectroscopy, to the realization of quantum gates in quantum information science. The experimental part of this research is often very delicate and expensive, and control theory undoubtedly can bring new prospectives to it.

In the present paper we deal with dissipative quantum control systems. The recent interest for such systems comes from several applications. The model problem we analyze below is related to the molecular alignment in the gas phase by a laser field, and to the control of the dynamics of spin $1 / 2$ particles in the liquid phase using nuclear magnetic resonance (NMR) with possible applications in medical imaging.

We consider a control system of the form

$$
\left\{\begin{array}{l}
\dot{x}=-\Gamma x+u_{2} z, \\
\dot{y}=-\Gamma y-u_{1} z, \\
\dot{z}=\gamma_{-}-\gamma_{+} z+u_{1} y-u_{2} x,
\end{array}\right.
$$

Translated from Sovremennaya Matematika i Ee Prilozheniya (Contemporary Mathematics and Its Applications), Vol. 82, Nonlinear Control and Singularities, 2012. 
where $\Gamma, \gamma_{-}$, and $\gamma_{+}$are some physical constants. Equations (1) are called the Kossakowsky-Lindblad equations, they describe the behavior of a two-level quantum system in a dissipative environment. The components of the state $q=(x, y, z) \in \mathbb{R}^{3}$ are related to the components of the density operator of the quantum system [7], while $\Gamma, \gamma_{+}$, and $\gamma_{-}$are the parameters describing the dissipation effect. The control is represented by the electromagnetic field $\left(u_{1}, u_{2}\right)$. By assumption, the dissipation parameters verify the constraint

$$
2 \Gamma \geq \gamma_{+} \geq\left|\gamma_{-}\right|,
$$

which guarantees that the unit Bloch ball $\|q\| \leq 1$ is invariant for the dynamics of (1). The point $\left(0, \gamma_{-} / \gamma_{+}\right)$is the equilibrium of the control-free system with $u_{1}=u_{2}=0$.

In the NMR context, Eqs. (1) are known as the Bloch equations describing the controlled dynamics of a spin $1 / 2$ particle. The duality between the two physical problems is very important since the NMR experiments are much easier to realize. The standard form of the Bloch equations is the following:

$$
\left\{\begin{array}{l}
\dot{M}_{x}=-\frac{1}{T_{2}} M_{x}+\omega_{y} M_{z} \\
\dot{M}_{y}=-\frac{1}{T_{2}} M_{y}-\omega_{x} M_{z}, \\
\dot{M}_{z}=-\frac{1}{T_{1}}\left(M_{0}-M_{z}\right)+\omega_{x} M_{y}-\omega_{y} M_{x} .
\end{array}\right.
$$

Here $\vec{M}=\left(M_{x}, M_{y}, M_{z}\right)$ is the total magnetization vector to which one applies the controlled magnetic field $\left(\omega_{x}, \omega_{y}, 0\right), T_{1}$ and $T_{2}$ are the relaxation times, and $\left(0,0, M_{0}\right)^{T}$ is the thermal equilibrium of the system. It is not difficult to see that by a proper reparametrization, (2) can be transformed into a system of the form (1), where the set of dissipation parameters is restricted to the subset $\gamma_{-}=\gamma_{+}$. In this case, the control-free equilibrium coincides with the north pole of the Bloch sphere $\|q\|=1$. More generally, NMR control systems can be obtained by coupling such spin 1/2 particles (with different parameters) controlled by the same magnetic field.

In the present paper we study the energy minimization problem for (1), which is formulated as follows: given a fixed terminal time $t_{f}>0$ and extremity points $q(0)=q_{0}, q\left(t_{f}\right)=q_{1}$, we search for the solutions of (1) minimizing the energy of the system:

$$
\frac{1}{2} \int_{0}^{t_{f}}\left(u_{1}^{2}(t)+u_{2}^{2}(t)\right) d t \rightarrow \min .
$$

The dynamics of the control problem under consideration can be rewritten in the form $\dot{q}=F_{0}(q)+$ $u_{1} F_{1}(q)+u_{2} F_{2}(q)$, where $F_{i}, i=0,1,2$, are smooth vector fields on $\mathbb{R}^{3}$. According to the Pontryagin maximum principle, the minimizers of the problem can be selected among the extremal curvessolutions to the equations

$$
\frac{d q}{d t}=\frac{\partial H}{\partial p}, \quad \frac{d p}{d t}=-\frac{\partial H}{\partial q}, \quad \frac{\partial H}{\partial u}=0,
$$

associated to the Hamiltonian function

$$
H(p, q, u)=H_{0}(p, q)+u_{1} H_{1}(p, q)+u_{2} H(p, q)+p_{0}\left(u_{1}^{2}+u_{2}^{2}\right),
$$

where $p_{0} \leq 0$ and $H_{i}(p, q)=\left\langle p, F_{i}(q)\right\rangle, i=0,1,2$, are the Hamiltonian lifts of the vector fields $F_{i}$. The extremal curves split into two categories that can be easily calculated:

- Normal case: $p_{0}=-1 / 2$. Then the last equation of (4) yields $u_{i}=H_{i}, i=1,2$, and plugging such a control into $H$, we obtain a smooth Hamiltonian function

$$
H_{n}=H_{0}+\frac{1}{2}\left(H_{1}^{2}+H_{2}^{2}\right) .
$$


The corresponding extremals are called normal. Their structure becomes clearer if we pass to the spherical coordinates

$$
x=\rho \sin \varphi \cos \theta, \quad y=\rho \sin \varphi \sin \theta, \quad z=\rho \cos \varphi,
$$

which better highlight the symmetry of revolution of (1). In new coordinates the normal Hamiltonian takes the form:

$H_{n}=p_{\rho}\left(\gamma_{-} \cos \varphi-\rho\left(\delta \cos ^{2} \varphi+\Gamma\right)\right)+p_{\varphi}\left(-\frac{\gamma_{-}}{\rho} \sin \varphi+\delta \sin \varphi \cos \varphi\right)+\frac{1}{2}\left(p_{\varphi}^{2}+p_{\theta}^{2} \cot ^{2} \varphi\right)$,

where $\delta=\gamma_{+}-\Gamma$.

- Abnormal case: $p_{0}=0$. In this case, the last equation of (4) yields the following conditions: $H_{1}(p(t), q(t))=H_{2}(p(t), q(t))=0$ for almost all $t \in\left[0, t_{f}\right]$. The abnormal control can be computed by further differentiation of these conditions with respect to time.

In our recent paper [5] we showed that due to the rotational symmetry of problem (1), (3), one must distinguish between 2D and 3D normal trajectories. In addition, if $\gamma_{-}=0$, the normal Hamiltonian describes a system integrable in quadratures, whose solutions can be computed explicitly in terms of elliptic functions. Moreover, if $\Gamma-\gamma_{+}=0$, then the resulting Hamiltonian system describes the geodesics of a particular almost-Riemannian metric on the 2D sphere of revolution having singularity on the equator, called Grushin's metric. Metrics of this type were widely studied in the framework of geometric control (see, e.g., $[2,4]$ ).

The observations above suggest describing the dissipation effect through a comparison of the solution of the problem (1), (3) in the general case with the geodesics of the Grushin metric. In a recent paper [6], Bonnard, Caillau, and Cots performed an accurate numerical analysis of the conjugate and cut loci for one particular class of the solutions of (1), (3), whose radial component is not controllable, and the study reduces to the analysis of a certain deformation of Grushin's metric on a 2D sphere of revolution. In contrast, this paper focuses on the flat $2 \mathrm{D}$ trajectories lying in the meridian planes of the Bloch ball. Improving our results from [5], we present here new analytical estimates for the conjugate and cut times for one particular class of flat trajectories. Then we compute numerically the optimal synthesis of the $2 \mathrm{D}$ integrable problem. It is worth nothing that the $2 \mathrm{D}$ problem is of a particular interest in the NMR context, where it is related to the so-called demagnetization problem with the initial state $q_{0}$ fixed in the north pole of the Bloch ball.

This paper is organized as follows. In the first two sections we briefly recall the main facts concerning abnormal and normal extremals of the energy minimization problem for (1) in the general 3D case. In particular, we reduce our problem to the analysis of a natural mechanical system, which allows us to classify and to compute explicitly the extremals in the integrable case. In Sec. 5 we derive an implicit equation that describes the conjugate points along one particular class of $2 \mathrm{D}$ normal extremals and provide analytical estimates for its solutions. We use these estimates in Sec. 6, where we present our numerical results concerning the structure of cut and conjugate loci of the $2 \mathrm{D}$ integrable problem using the Hampath algorithm [8]. In Sec. 7 we discuss the nonintegrable case. Finally, in the Appendix we list the explicit parametrization formulas for the normal extremals.

\section{Abnormal Extremals in the Energy Minimization Problem}

As we showed in [5], the study of the abnormal extremals of (1), (3) can be restricted to the analysis of the abnormal extremals of a $2 \mathrm{D}$ single-input control system $\dot{q}=F_{0}(q)+u_{1} F_{1}(q)$, where $q=(y, z)$. The structure of the abnormal flow of $2 \mathrm{D}$ systems is well understood (see for instance, [3]): they are contained in the set $S=\left\{q: \operatorname{det}\left(F_{0},\left[F_{0}, F_{1}\right]\right)\right\}=0$, while the abnormal control can be computed as 
follows:

$$
u_{a}=-\frac{\left\langle p,\left[\left[F_{1}, F_{0}\right], F_{0}\right](q)\right\rangle}{\left\langle p,\left[\left[F_{1}, F_{0}\right], F_{1}\right](q)\right\rangle} .
$$

The adjoined variable $p$ can be then eliminated using the condition $\left\langle p, F_{1}(q)\right\rangle=0$.

Another important set in our problem is the collinear set

$$
C=\left\{q: \operatorname{det}\left(F_{0}(q), F_{1}(q)\right)=0\right\} .
$$

This set has the form of an oval, symmetric with respect to the $z$-axis, which passes through the origin and through the control-free equilibrium $\left(0, \gamma_{-} / \gamma_{+}\right)$on the $y z$-plane. If $\gamma_{-}=0$, the collinear set shrinks to the origin.

Direct computation yields the following result [5].

Proposition 1. If $\delta=\gamma_{+}-\Gamma \neq 0$, the abnormal curves form two lines:

(1) the $z$-axis of revolution $y=0$, the corresponding abnormal control being $u_{a}=0$;

(2) the abnormal horizontal line $z=\frac{\gamma_{-}}{2 \delta}$, the abnormal control being

$$
u_{a}=\frac{\gamma_{-}}{2 y \delta}\left(\gamma_{+}-2 \Gamma\right)
$$

which blows up at $y=0$ if $\gamma_{-}\left(\gamma_{+}-2 \Gamma\right) \neq 0$. In particular, in this case the abnormal control is in the $L^{1}$, but not in the $L^{2}$ category next to $y=0$. If $\gamma_{-}=0$, the abnormal control is 0 .

Concerning the role of the abnormal lines in the energy minimization problem, one has the following theorem.

Theorem 1. Let $\gamma: t \in\left[0, t_{f}\right] \rightarrow \gamma(t)$ be any sub-arc of the horizontal or vertical abnormal arc such that $t \mapsto \gamma(t)$ is one-to-one, and consider the extremity $A=\gamma\left(t_{f}\right)$. Then the abnormal control is the only control steering $\gamma(0)$ to $A$ in a time $t_{f}$, provided the trajectory remains in a $C^{0}$-tube around $\gamma(\cdot)$. For the energy minimization problem, such an arc is optimal in the described neighborhood, whereas any broken abnormal trajectory formed by a concatenation of abnormal horizontal and vertical arcs is not optimal.

\section{Normal Case}

First, observe that the symmetry of (1) and of the cost functional with respect to the rotations about $z$-axis leads to the following result.

Proposition 2. Consider a Hamiltonian system associated to the normal Hamiltonian $H_{n}$. Then

(1) $\theta$ is a cyclic variable and the corresponding canonical impulse $p_{\theta}$ is a first integral along normal extremals;

(2) if $\gamma_{-}=0$, then the normal Hamiltonian flow is Liouville integrable, the additional first integral being $p_{r}$, where $r=\ln \rho$. In this case, the corresponding Hamiltonian is given by

$$
H_{n}=-p_{r}\left(\delta \cos ^{2} \varphi+\Gamma\right)+\delta p_{\varphi} \sin \varphi \cos \varphi+\frac{1}{2}\left(p_{\varphi}^{2}+p_{\theta}^{2} \cot ^{2} \varphi\right) .
$$

Since $\dot{\theta}=p_{\theta} \cot ^{2} \theta$, the proposition above means that the projections on the state space of the normal extremals lying on the level set $p_{\theta}=0$ are contained in a fixed meridian plane $\theta=$ const of the Bloch ball. Moreover, this property is shared by all normal trajectories starting from the $z$-axis since $p_{\theta}=x p_{y}-y p_{x}$. Thus, as in the abnormal case, if $p_{\theta}=0$, the problem can be reduced to a $2 \mathrm{D}$ single input control problem in the plane $y z$ (with $u_{2}=0$ ).

A key property in the analysis of the energy minimization problem for (1) is the introduction of a natural mechanical system. Indeed, the particular form of the Hamiltonian $H_{n}$ implies the following proposition. 
Proposition 3. The equation $H_{n}=h$ can be written as follows:

$$
\frac{1}{2} \dot{\varphi}^{2}+V\left(\varphi, \rho, p_{\rho}\right)=h,
$$

where

$$
V\left(\varphi, \rho, p_{\rho}\right)=\gamma_{-} p_{\rho} \cos \varphi-\rho p_{\rho}\left(\delta \cos ^{2} \varphi+\Gamma\right)-\frac{1}{2}\left(\delta \frac{\sin (2 \varphi)}{2}-\frac{\gamma_{-} \sin \varphi}{\rho}\right)^{2}+\frac{1}{2} p_{\theta}^{2} \cot ^{2} \varphi
$$

is a potential energy.

As a direct consequence of Propositions 2 and 3 we obtain the following proposition.

Proposition 4. If $\gamma_{-}=0$, the energy minimization problem for (1) is integrable in quadratures. More precisely, the evolution of the $\varphi$ variable is described by the natural mechanical system

$$
\frac{\dot{\varphi}^{2}}{2}+V(\varphi)=h
$$

where

$$
V(\varphi)=-p_{r}\left(\delta \cos ^{2} \varphi+\Gamma\right)-\frac{\delta^{2}}{8} \sin ^{2} 2 \varphi+\frac{1}{2} p_{\theta}^{2} \cot ^{2} \varphi .
$$

The remaining variables can be found from the equations

$$
\dot{\theta}=p_{\theta}\left(\operatorname{cosec}^{2} \varphi-1\right), \quad \dot{r}=\delta \sin ^{2} \varphi-\gamma_{+} .
$$

If $\gamma_{-}=0$, a special case occurs for $\delta=\gamma_{+}-\Gamma=0$ : the $\rho$-variable cannot be controlled and the energy minimization problem is equivalent to the length minimization problem for the metric $g=d \varphi^{2}+\tan ^{2} \varphi d \theta^{2}$ on a $2 \mathrm{D}$ sphere of revolution. This metric appears also in the time-minimal control problem, since if we parameterize it by the arc-length, the length corresponds to the time.

Definition 1. The quasi-Riemannian metric $g=d \varphi^{2}+\tan ^{2} \varphi d \theta^{2}$ with a singularity on the equator is called the standard Grushin metric on the two-sphere of revolution.

A detailed analysis of Grushin's metric can be found, for example, in [4]. The Grushin geodesics are either meridian circles or periodic trajectories in the plane $\left(\varphi, p_{\varphi}\right)$ with $\dot{\theta}$ periodic. The associated phase-portrait has the same structure as the right column in Fig. 1. The cut and conjugate loci corresponding to the point $(\varphi(0), \theta(0))$, where $\theta(0)$ can be set at 0 , are as follows:

- $\phi(0)=0$ or $\pi$. In this case, $q_{0}$ is a pole and the conjugate and cut loci are formed by the antipodal pole;

- $\phi(0) \neq k \pi / 2, k=0,1, \ldots$ If $q_{0}$ is neither a pole nor a point on the equator, the cut locus is a segment of the antipodal parallel, while the conjugate locus has the shape of an astroid with four cusps. The distance to the cut locus can be easily computed: it is the length of the unique geodesic starting tangentially to the parallel and reaching the antipodal parallel in a tangent way.

- $\phi(0)=\pi / 2$. In this case, the cut locus is the whole equator minus the initial point, while the conjugate points accumulate at $\phi(0)$. Hence the distance to the cut locus is zero.

The deformation of the Grushin metric caused by the dissipation when $\delta \neq 0$ generates quite an intricate dynamics inside the Bloch sphere, which can be explicitly computed in the integrable case $\gamma_{-}=0$. 


\section{Integrable Case}

4.1. Classification of normal extremals. In order to obtain an explicit parametrization of the solutions of (7), we reduce the computations to standard elliptic integrals. For this, let us introduce an auxiliary variable $x=\sin ^{2} \varphi$, and rewrite (7) in the following way:

$$
\frac{\dot{\varphi}^{2}}{2}+\bar{W}(\varphi)=\bar{h}
$$

where $\bar{h}=h+p_{r} \gamma_{+}+\frac{p_{\theta}^{2}}{2}$ is constant, $\bar{W}(\varphi)=W(x(\varphi))$ and

$$
W(x)=\frac{\delta^{2}}{2 x}\left(x^{3}-2 a x^{2}+\frac{p_{\theta}^{2}}{\delta^{2}}\right), \quad a=\frac{1}{2}-\frac{p_{r}}{\delta} .
$$

Observe that (9) is invariant with respect to the reflections about the vertical axis $\varphi \rightarrow-\varphi$ and to the shifting $\varphi \rightarrow \varphi+\pi$. Our further analysis is based on the elementary properties of the function $\bar{W}$ for $\varphi \in[0, \pi]$. It is easy to see that (see [5] for details) if $p_{\theta} \neq 0$, then $\bar{W}(\varphi) \rightarrow+\infty$ as $\varphi \rightarrow 0 \bmod \pi$, $\bar{W}(\pi / 2)=\delta p_{r}+p_{\theta}^{2} / 2$, and it can have at most two critical points where it attains its minimum. In contrast, when $p_{\theta}=0$, then $\bar{W}$ is bounded: it has two local maxima at $\varphi=0, \pi / 2, \pi$ and $\min _{[0, \pi]} \bar{W}(\varphi)=$ $\min \left\{\delta p_{r},-\frac{a^{2} \delta^{2}}{2}\right\}, \max _{[0, \pi]} \bar{W}(\varphi)=\max \left\{\delta p_{r}, 0\right\}$.

In Figs. 1-3 we list all possible forms of $\bar{W}$ and present the corresponding phase-portraits for the solutions of (9). There are three topologically different types of trajectories on the $(\varphi, \dot{\varphi})$ plane:

- oscillating trajectories, which are homotopic to zero;

- rotation trajectories, which are periodic trajectories not homotopic to zero;

- nonperiodic trajectories corresponding to the separatrices in Fig. 1-3.

Rotation trajectories correspond to the meridian circles in the Grushin case, which are the only class of flat geodesics of this metric. In our case the two new types of motion appear due to the influence of the dissipation terms.

In what follows, among the oscillating trajectories we will distinguish between

(1) parallel orbits: $\varphi=$ const, corresponding to the critical points of the potential $\bar{W}$;

(2) short periodic orbits. For certain values of the dissipation parameters and of the constants of motion, there can appear a pair of families of periodic orbits characterized by the following property: $\varphi(t) \in\left[\varphi_{\min }, \varphi_{\max }\right]$ and $\varphi_{\max }-\varphi_{\min }<\pi / 2$. In other words, these orbits remain in the north or in the south semi-spheres of the Bloch ball and never cross the equatorial plane;

(3) long periodic orbits are periodic orbits such that $\frac{\pi}{2}<\varphi_{\max }-\varphi_{\min }<\pi$. If $p_{\theta} \neq 0$ or $p_{\theta}=0$ and $p_{r} \delta<0$ they cross the equatorial plane, whereas in the case $p_{\theta}=0$ and $p_{r} \delta>0$ they cross the $z$-axis.

4.2. Integration in terms of Jacobi elliptic functions. An explicit parametrization of $\varphi(t)$ can be obtained in the following way. From (9) we obtain

$$
\dot{\varphi}= \pm \sqrt{2(\bar{H}-W(x(\varphi)))} .
$$

Let us take the positive branch, the other case being equivalent modulo inversion of time. Passing to $x=\sin ^{2} \varphi$, we obtain

$$
d t=\frac{d x}{2 \sqrt{2} \sqrt{x(1-x)(\bar{h}-W(x))}} .
$$




$$
p_{\theta}^{2}<\delta^{2}+2 \delta p_{r}
$$
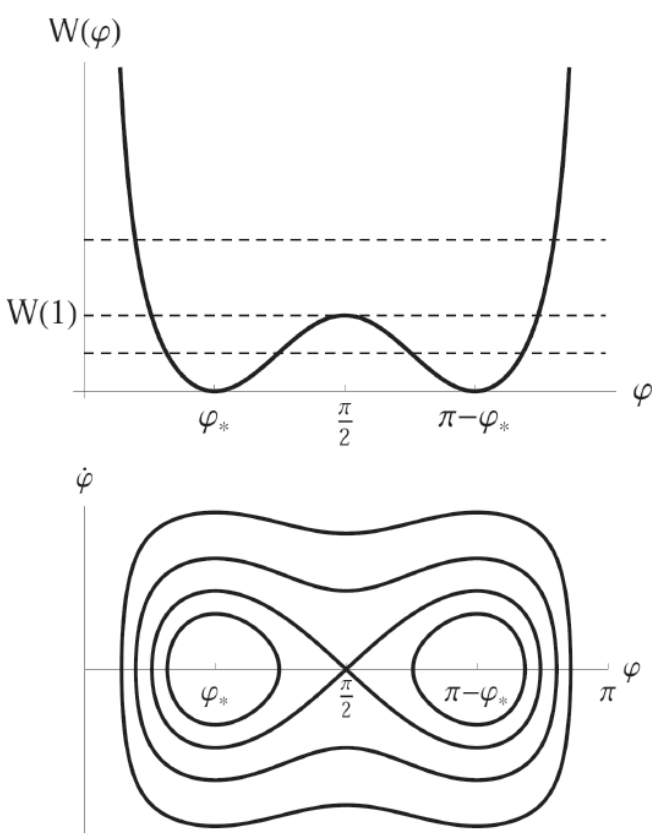

$p_{\theta}^{2} \geq \delta^{2}+2 \delta p_{r}$
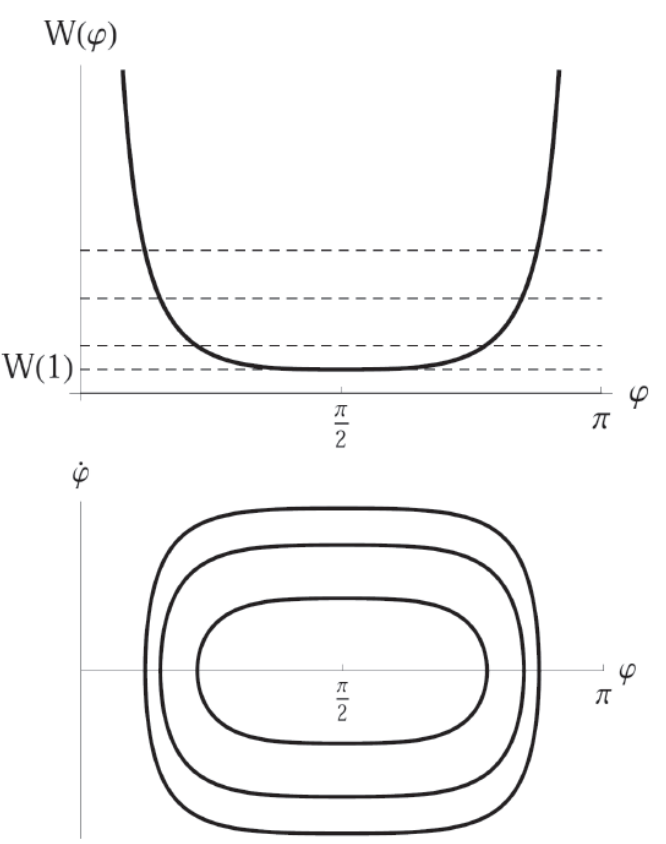

Fig. 1. 3D case: $p_{\theta} \neq 0$.

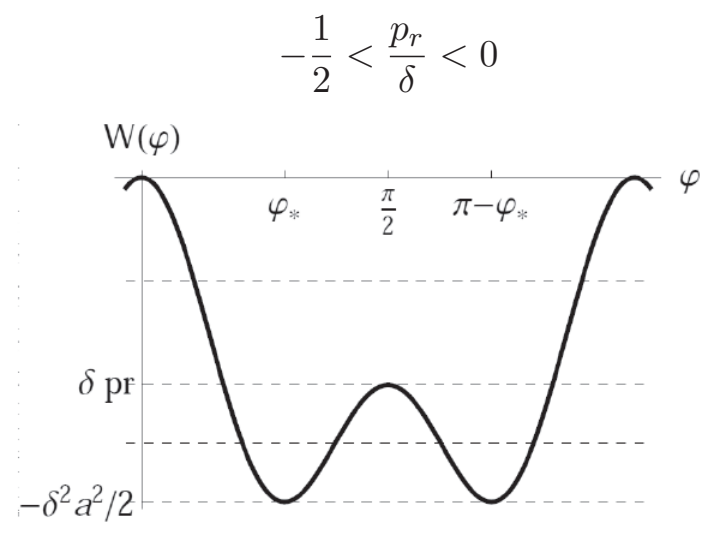

$$
0<\frac{p_{r}}{\delta}<\frac{1}{2}
$$
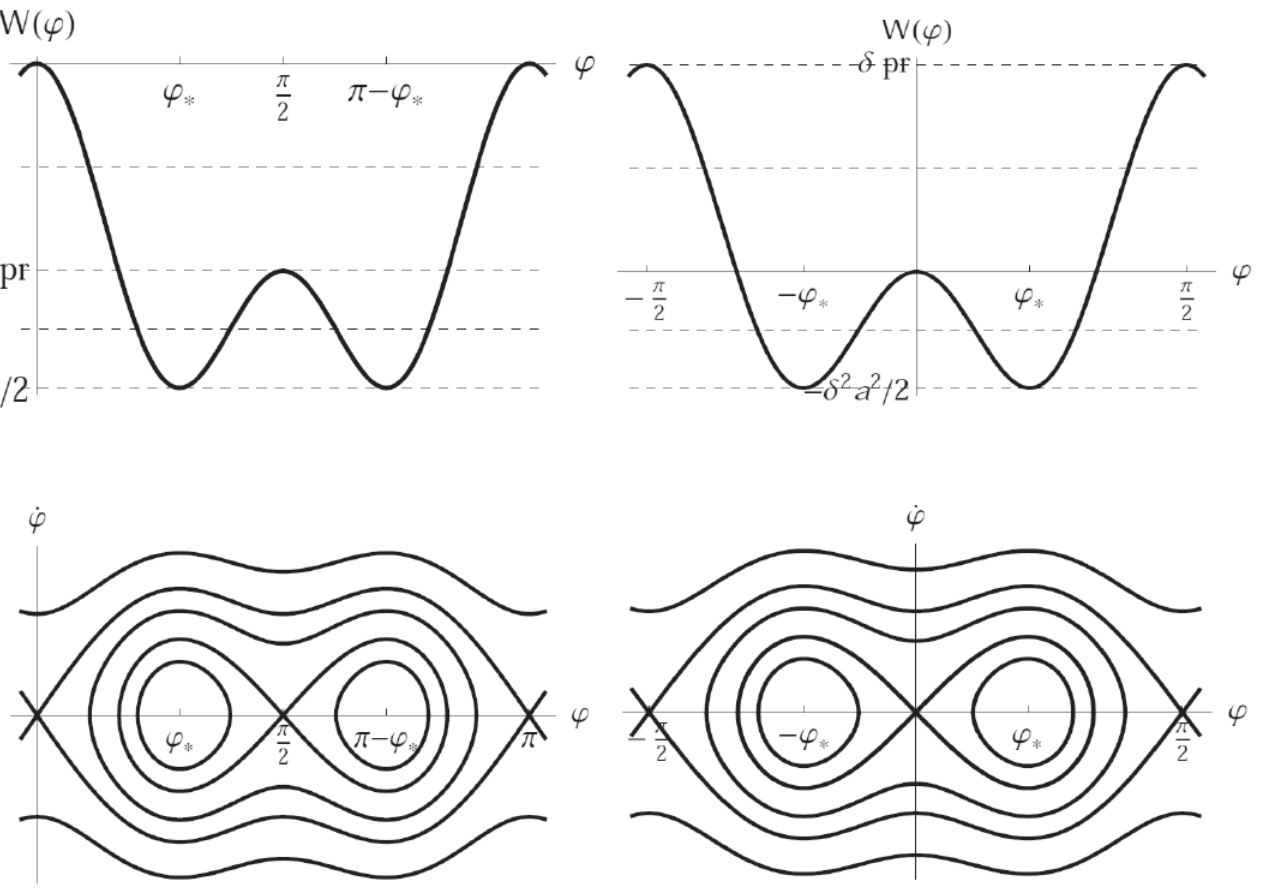

Fig. 2. 2D case: $\left.p_{\theta}=0, a \in\right] 0,1[$. 


$$
\frac{p_{r}}{\delta} \leq-\frac{1}{2}
$$
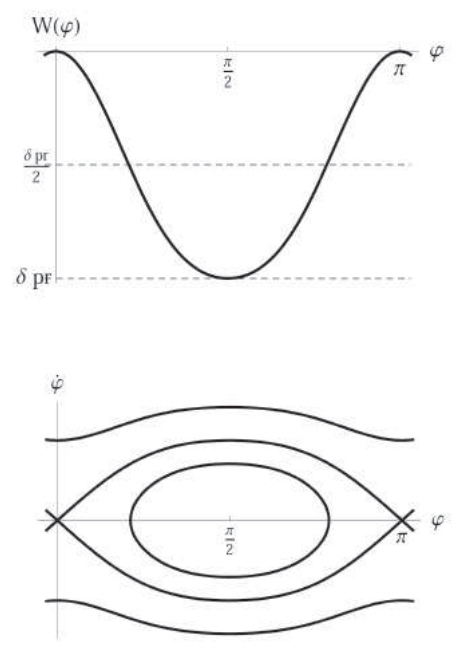

$p_{r}=0$
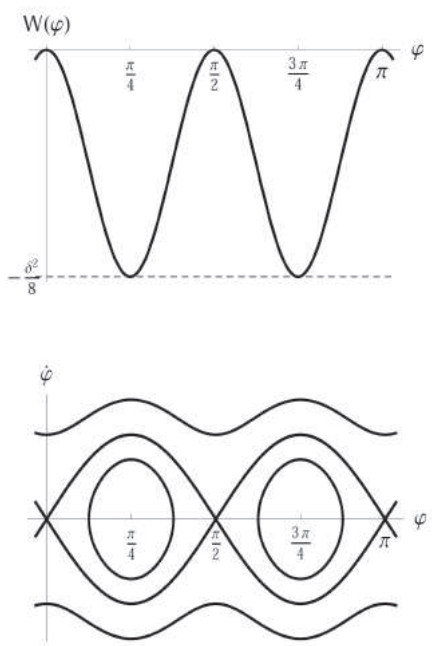

$$
\frac{p_{r}}{\delta} \geq \frac{1}{2}
$$
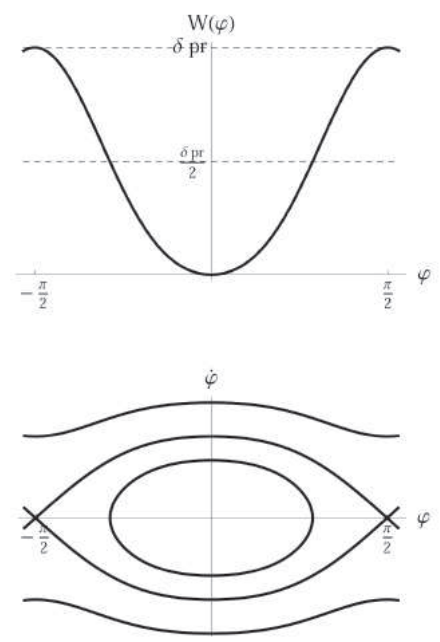

Fig. 3. 2D case: $\left.p_{\theta}=0, a \notin\right] 0,1[$.

Further calculation is related to the analysis of the roots of the cubic polynomial

$$
P(x)=x(\bar{h}-W(x))=x^{3}-2 a x^{2}-\frac{2 \bar{h}}{\delta^{2}} x+\frac{p_{\theta}^{2}}{\delta^{2}} .
$$

It is easy to show (see [5] for the details) that if $p_{\theta} \neq 0, P$ has three real roots: $x_{3}<0$, and two positive roots $0<x_{1} \leq x_{2}$. If $p_{\theta}=0$, then $x_{3}=0, x_{1}<x_{2}$, and $x_{1}$ can be of both signs. Since $x=\sin ^{2} \varphi$, the final type of parametrization depends on the placement of $x_{1}$ and $x_{2}$ with respect to the interval $[0,1]$.

Assume that $y_{i} \in\left\{x_{1}, x_{2}, x_{3}, 1\right\}, i=1,2,3,4$, are ordered is such a way that $y_{1}>y_{2}>y_{3}>y_{4}$. Then

$$
\frac{d x}{d t}=\sqrt{\delta^{2}\left(x-y_{1}\right)\left(x-y_{2}\right)\left(x-y_{3}\right)\left(x-y_{4}\right)} .
$$

Following the method proposed in [10], we set

$$
z^{2}=\frac{\left(y_{2}-y_{4}\right)\left(x-y_{3}\right)}{\left(y_{2}-y_{3}\right)\left(x-y_{4}\right)}
$$

Then

where

$$
\frac{1}{\Delta(z)} \frac{d z}{d t}=\frac{M}{\Delta_{1}(x)} \frac{d x}{d t}
$$

$$
\begin{gathered}
\Delta^{2}(z)=\left(1-z^{2}\right)\left(1-k^{2} z^{2}\right), \quad \Delta_{1}^{2}(x)=4\left(x-y_{1}\right)\left(x-y_{2}\right)\left(x-y_{3}\right)\left(x-y_{4}\right), \\
M^{2}=\delta^{2}\left(y_{1}-y_{3}\right)\left(y_{2}-y_{4}\right), \quad k^{2}=\frac{\left(y_{2}-y_{3}\right)\left(y_{1}-y_{4}\right)}{\left(y_{1}-y_{3}\right)\left(y_{2}-y_{4}\right)} .
\end{gathered}
$$

The chosen order of the roots $y_{i}$ assures $k \in(0,1)$, and $z$ can be computed from a standard elliptic integral, which yields

$$
z(t)=\operatorname{sn}\left(M t+\psi_{0}, k\right), \quad \psi_{0}=\mathrm{sn}^{-1}(z(0), k) .
$$


In particular, $z(t)$ is a periodic function, which oscillates between -1 and 1 with the period $4 K(k) / M$, where $K(k)$ denotes the complete elliptic integral of the first kind of modulus $k$.

Finally, by inverting the Möbius transformation we obtain

$$
x(t)=\frac{z^{2}(t)\left(y_{2}-y_{3}\right) y_{4}-\left(y_{2}-y_{4}\right) y_{3}}{z^{2}(t)\left(y_{2}-y_{3}\right)-\left(y_{2}-y_{4}\right)} .
$$

The original variable $\varphi$ can be then computed taking, if needed, either $\arcsin \sqrt{x(t)}$ or $\pi-\arcsin \sqrt{x(t)}$ in order to get an analytic function.

The integration of the remaining variables $\theta$ and $r$ reduces to the computation of elliptic integrals of the third kind. We omit here the technical details and present directly the result:

$$
\begin{aligned}
r(t)-r(0)= & \left(\delta y_{4}-\gamma_{+}\right) t+\frac{\left(y_{3}-y_{4}\right) \delta}{M}\left(\Pi\left[\frac{y_{2}-y_{3}}{y_{2}-y_{4}}, \operatorname{am}\left(M t+\psi_{0}, k\right), k\right]\right. \\
& \left.-\Pi\left[\frac{y_{2}-y_{3}}{y_{2}-y_{4}}, \operatorname{am}\left(\psi_{0}, k\right), k\right]\right), \\
\theta(t)-\theta(0)= & \frac{p_{\theta}\left(1-y_{4}\right) t}{y_{4}}-\frac{p_{\theta}\left(y_{3}-y_{4}\right)}{M y_{3} y_{4}}\left(\Pi\left[\frac{y_{4}\left(y_{2}-y_{3}\right)}{y_{3}\left(y_{2}-y_{4}\right)}, \operatorname{am}\left(M t+\psi_{0}, k\right), k\right]\right. \\
& \left.-\Pi\left[\frac{y_{4}\left(y_{2}-y_{3}\right)}{y_{3}\left(y_{2}-y_{4}\right)}, \operatorname{am}\left(\psi_{0}, k\right), k\right]\right) .
\end{aligned}
$$

The final formulas for each particular case listed in Figs. 1-3 depend on the placement of the roots $x_{1,2}$ with respect to 0 and 1 , and we list them in the Appendix.

4.3. Symmetries and Optimality. Before discussing the optimality properties of the extremal solutions, let us recall some standard definitions from Riemannian geometry [9].

Definition 2. Denote by $\vec{H}_{n}$ the Hamiltonian vector field of the normal Hamiltonian $H_{n}$ and let $\exp \left[t \vec{H}_{n}\right]$ denote the associated Hamiltonian flow. Let $z(t)=(p(t), q(t)), t \in\left[0, t_{f}\right]$ be a reference extremal.

(1) For fixed $q(0)=q_{0}$, the map

$$
\exp _{q_{0}, t}: p(0) \mapsto \Pi\left[\exp t \vec{H}_{n}\left(q_{0}, p(0)\right)\right],
$$

where $\Pi:(p, q) \mapsto q$ is a standard projection, is called the end-point mapping of the flow $\exp \left[t \vec{H}_{n}\right]$. The time $t_{*}$ is said to be conjugate to $t=0$ if $\exp _{q_{0}, t_{*}}$ is not an immersion. We denote by $t_{*}^{1}$ the first conjugate time and the corresponding first conjugate point by $q\left(t_{*}^{1}\right)$.

(2) The point $q\left(t_{s}\right)$ on the reference extremal is a separating point if there exists another extremal curve $z^{\prime}(\cdot)=\left(p^{\prime}(\cdot), q^{\prime}(\cdot)\right)$ with distinct $q(\cdot)$ and $q^{\prime}(\cdot)$, such that $q\left(t_{s}\right)=q^{\prime}\left(t_{s}\right)$ and $q$ and $q^{\prime}$ have the same cost at $t_{s}$.

(3) The cut-point along the reference extremal is the first point $q\left(t_{c}\right)$ such that $q(\cdot)$ is no longer optimal beyond the time $t_{c}$. Fixing the final time to $t_{f}$, we see that the set of such points form respectively the conjugate locus $C\left(q_{0}\right)$, the separating locus $L\left(q_{0}\right)$, and the cut locus $C_{u t}\left(q_{0}\right)$.

Our next goal is to investigate the optimality of normal extremals in the integrable case. Taking into account the action of the discrete symmetric group on the set of extremals, we can immediately compute obvious separating points. Indeed, fixing $p_{\theta}$ and $p_{r}$, for each initial condition $\varphi(0)$ we have two extremal curves on the level set $\bar{h}$, starting in opposite directions defined by $\pm \dot{\varphi}(0)$. These curves are distinct and periodic (in $\varphi$ variable) provided none of the following cases is realized: $\varphi(0)$ is an equilibrium point, $\dot{\varphi}(0)=0$, or the level set $\bar{h}$ carries on rotations (for the fixed values of $p_{r}$ and $p_{\theta}$ ). If $T$ is the corresponding period of $\varphi$, we immediately deduce the following proposition. 


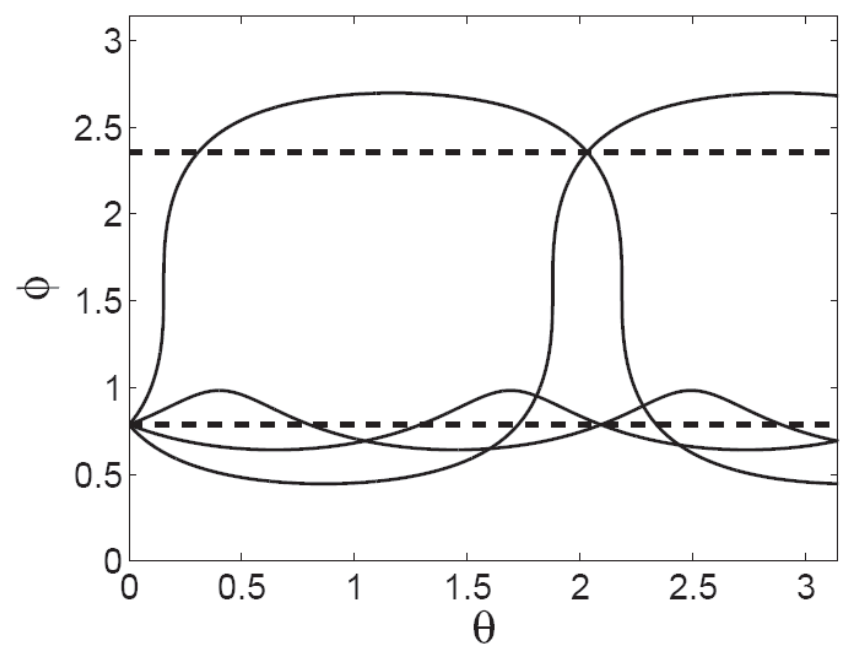

Fig. 4. Long and short normal extremals in the 3D case and the corresponding separation points.

Proposition 5. For fixed $p_{r}$ and $p_{\theta}$, the two distinct periodic extremal curves starting from $\varphi(0)$ with the same $\theta(0)$ and $r(0)$ intersect at the same point with the same cost after one period $T$. Hence the corresponding point belongs to the separating locus.

Taking into account the central symmetry of the long extremals, we have the following proposition.

Proposition 6. Two distinct long periodic extremals starting at the same point $q_{0}$ intersect after an half-period T/2 with the same cost, and hence the associated point belongs to the separating locus.

Proof. For long periodic extremals, one can use the property that system (9) and the cost are reflexionally symmetric with respect to the equator. Hence, both curves starting from $\dot{\varphi}(0)$ and $-\dot{\varphi}(0)$ intersect on the antipodal parallel $\pi-\varphi(0)$ at the time $T / 2$ and with the same cost. This is also true for the $\theta$ and $r$ components. The same argument remains true for $2 \mathrm{D}$ long extremals symmetric with respect to $\varphi=0$ for $\delta p_{r}>0$ : after time $T / 2$ they intersect at $-\varphi(0)$.

The analysis of conjugate and cut loci in the general 3D integrable case is far from being complete, and there are only some partial numerical results for it. But in two particular cases corresponding to zero values of the first integrals $p_{r}$ and $p_{\theta}$ the study can be reduced to a $2 \mathrm{D}$ situation.

- Spherical case. If $p_{r}=0$, the variable $r$ is not controllable. The analysis can be reduced to the study of the projections of the optimal trajectories on the Bloch sphere endowed with a Lorenzian metric defined by the Hamiltonian $H_{n}$. This metric can be seen as a deformation of the Grushin metric on a 2D sphere of revolution appearing if $\delta=0$. In the recent paper [6] J. B. Caillau and the first two authors provided a detailed numerical analysis of the optimal synthesis in the spherical case.

- Meridian case. As we saw above, if $p_{\theta}=0$, the normal trajectories are flat and the physical motion takes place in a fixed meridian plane $\theta_{0}$ in the Bloch ball.

In the remaining part of this paper we investigate the structure of conjugate and cut points of $2 \mathrm{D}$ normal extremals in the meridian planes. 


\section{Meridian Case $p_{\theta}=0$}

5.1. Geometrical structure of normal extremals in $2 \mathrm{D}$ case. According to Figs. 2 and 3 , in the meridian case we have:

- Parallel solutions, which in the 2D flat case become straight lines on the $y z$-plane. For all values of $p_{r}$ on the level sets $\bar{h}=\delta p_{r}$ and $\bar{h}=0$ we have equilibria $\varphi=0$ and $\varphi=\frac{\pi}{2} \bmod \pi$, which results in horizontal and vertical axes on the $y z$-plane. These normal trajectories project on the abnormal ones. In addition, if

$$
\frac{\left|p_{r}\right|}{|\delta|}<\frac{1}{2}
$$

on the level set $\bar{h}=-\frac{\delta^{2} a^{2}}{2}$ we have a couple of equilibria at $\varphi_{*}= \pm \arcsin \sqrt{a} \bmod \pi$, which define a straight line in each quadrant of the $y z$-plane. Note that

$$
\varphi_{*}=\frac{\pi}{4} \bmod \frac{\pi}{2}
$$

if $p_{r}=0$.

- Short orbits exist if

$$
\frac{\left|p_{r}\right|}{|\delta|}<\frac{1}{2}
$$

on the level sets $-\frac{\delta^{2} a^{2}}{2}<\bar{h}<\delta p_{r}<0$ or $-\frac{\delta^{2} a^{2}}{2}<\bar{h}<0<\delta p_{r}$, depending on the sign of $\delta p_{r}$.

- Long orbits appear if $\delta p_{r}<\bar{h}<0$ or $0<\bar{h}<\delta p_{r}$. In the latter case they cross the vertical axis, but do not cross the horizontal axis $z=0\left(\varphi=\frac{\pi}{2} \bmod \pi\right)$.

- Rotations exist on the level sets $\bar{h}>\delta p_{r}>0$ and $\bar{h}<\delta p_{r}<0$.

- There could be one or two separatrices on the level sets $\bar{h}=0$ and $\bar{h}=\delta p_{r}$.

Note that in contrast to the 3D case (see Fig. 1), there are families of trajectories (rotations and long periodic trajectories for $\delta p_{r}>0$ ) having central symmetry with respect to 0 and crossing the vertical axis. Therefore, for any pair $\varphi_{1}, \varphi_{2} \in[0,2 \pi]$ on a proper level set $\bar{h}$ there exist a solution of (9) connecting them. These observations are important for the global optimality analysis that we discuss in the next section.

5.2. Conjugate points of short periodic orbits. According to the phase portraits classification, in the $2 \mathrm{D}$ case the short trajectories lie on the level sets

$$
-\frac{\delta^{2} a^{2}}{2}<\bar{h}<\delta p_{r}<0 \quad \text { or } \quad-\frac{\delta^{2} a^{2}}{2}<\bar{h}<0<\delta p_{r} .
$$

Consider a trajectory starting at $\varphi(0)=\varphi_{0}$ and $r(0)=r_{0}$. Denote $x_{0}=\sin ^{2} \varphi_{0}$,

$$
M=\sqrt{\delta^{2}\left(1-x_{1}\right) x_{2}}, \quad k^{2}=\frac{x_{2}-x_{1}}{x_{2}\left(1-x_{1}\right)}, \quad z_{0}^{2}=\frac{x_{2}\left(x_{0}-x_{1}\right)}{x_{0}\left(x_{2}-x_{1}\right.},
$$

where $x_{1}$ and $x_{2}$ are the roots of the quadratic equation $\bar{h}-W(x)=0$ :

$$
x_{1,2}=a \pm \sqrt{a^{2}+\frac{2 \bar{h}}{\delta^{2}}} .
$$

Along short orbits these roots satisfy

$$
0<x_{1} \leq x_{0} \leq x_{2}<1,
$$


and hence $k \in(0,1)$. Applying the formulas of Sec. 4.2, we obtain an explicit parametrization of short orbits in terms of Jacobi elliptic functions:

$$
\begin{aligned}
\varphi(t) & =\arcsin \sqrt{\frac{x_{1} x_{2}}{x_{2}-\left(x_{2}-x_{1}\right) \operatorname{sn}\left(M t+\psi_{0}, k\right)^{2}}}, \\
r(t)-r_{0} & =\frac{\delta x_{1}}{M}\left[\Pi\left(\frac{x_{2}-x_{1}}{x_{2}}, \operatorname{am}\left(M t+\psi_{0}, k\right), k\right)-\Pi\left(\frac{x_{2}-x_{1}}{x_{2}}, \operatorname{am}\left(\psi_{0}, k\right), k\right)\right]-\gamma_{+} t,
\end{aligned}
$$

where $\psi_{0}=\mathrm{sn}^{-1}\left(z_{0}, k\right)$. In particular, $\varphi(t)$ is a periodic function with period $\frac{2 K(k)}{M}$, where $K(k)$ denotes a complete elliptic integral of the first kind corresponding to the modulus $k$, which in the sequel we denote simply by $K$.

Consider now the endpoint mapping associated to our problem:

$$
\exp _{\left(\varphi_{0}, r_{0}\right), t}:\left(p_{\varphi}(0), p_{r}\right) \mapsto(\varphi(t), r(t)) .
$$

The extremals are parametrized by the initial values of the co-vector $\left(p_{\varphi}(0), p_{r}\right)$. To simplify calculations, let us first re-parametrize the extremals using $x_{1}$ and $m=k^{2}$. To this end we rewrite the end-point mapping as a composition $\exp _{\left(\varphi_{0}, r_{0}\right), t}=G \circ \Phi$, where

$$
\Phi:\left(p_{\varphi}(0), p_{r}\right) \mapsto\left(x_{1}, m\right), \quad G:\left(x_{1}, m\right) \mapsto(\varphi(t), r(t)) .
$$

It is easy to show that the map $\Phi$ is nondegenerate provided $\varphi_{0} \neq \arcsin \sqrt{x}_{i}, i=1,2$, or, equivalently, $\dot{\varphi}(0) \neq 0$. More precisely, a simple computation (see [5] for details) yields

$$
\operatorname{det} D_{\left(p_{\varphi}(0), p_{r}\right)} \Phi=\frac{2 x_{1}}{\delta^{3} x_{2}} \frac{p_{\varphi}(0)+\delta \sin \varphi_{0} \cos \varphi_{0}}{\sqrt{a^{2}+2 \frac{\bar{h}}{\delta^{2}}}}=\frac{4 x_{1} \dot{\varphi}(0)}{\delta^{3} x_{2}\left(x_{2}-x_{1}\right)} .
$$

Assume first $\varphi_{0} \neq \arcsin \sqrt{x_{1,2}}$, which implies $\psi_{0} \neq j K, j \in \mathbb{Z}$. Then the critical points of the end-point mapping are the critical points of $G$. We have

$$
D_{\left(x_{1}, m\right)} G=\frac{\partial \varphi}{\partial x} D_{\left(x_{1}, m\right)} G_{1}, \quad \text { where } \quad G_{1}:\left(x_{1}, m\right) \mapsto(x(t), r(t)),
$$

where $x=\sin ^{2} \varphi$. Since $x_{2}=\frac{x 1}{1-m(1-x 1)}$,

$$
x(t)=\bar{x}\left(z\left(t ; x_{1}, m\right) ; x_{1}, m\right)=\frac{x_{1}}{1-m\left(1-x_{1}\right) z(t)^{2}},
$$

where $^{1} z(t)=\operatorname{sn}\left(M t+\psi_{0} \mid m\right)$ with

$$
M=\left(\frac{\delta^{2} x_{1}\left(1-x_{1}\right)}{1-m\left(1-x_{1}\right)}\right)^{1 / 2}, \quad \operatorname{sn}\left(\psi_{0} \mid m\right)=z(0) .
$$

In particular, an easy computation shows that along short orbits $\frac{\partial \varphi}{\partial x} \neq 0$, and

$$
\Delta(t)=D_{\left(x_{1}, m\right)} G_{1}=\left(\begin{array}{cc}
\frac{\partial \bar{x}}{\partial z} \frac{\partial z(t)}{\partial x_{1}}+\frac{\partial \bar{x}}{\partial x_{1}} & \frac{\partial \bar{x}}{\partial z} \frac{\partial z(t)}{\partial m}+\frac{\partial \bar{x}}{\partial m} \\
\frac{\partial r(t)}{\partial x_{1}} & \frac{\partial r(t)}{\partial m}
\end{array}\right) .
$$

For brevity, below we write sn instead of $\operatorname{sn}\left(M t+\psi_{0} \mid m\right)$, and similarly for other elliptic functions. The direct calculation yields

$$
\frac{\partial \bar{x}}{\partial x_{1}}=\frac{\mathrm{dn}^{2}}{\left(1-m\left(1-x_{1}\right) \mathrm{sn}^{2}\right)^{2}}, \quad \frac{\partial \bar{x}}{\partial m}=\frac{x_{1}\left(1-x_{1}\right) \mathrm{sn}^{2}}{\left(1-m\left(1-x_{1}\right) \mathrm{sn}^{2}\right)^{2}}, \quad \frac{\partial \bar{x}}{\partial z}=\frac{2 m\left(1-x_{1}\right) x_{1} \mathrm{sn}}{1-m\left(1-x_{1}\right) \mathrm{sn}^{2}} .
$$

Hence

$$
\operatorname{det} \Delta(t)=\frac{\operatorname{det} \Delta_{1}(t)}{\left(1-m\left(1-x_{1}\right) \operatorname{sn}^{2}\right)^{2}},
$$

\footnotetext{
${ }^{1}$ Hereafter we use the notation of $[1]: \operatorname{sn}(y \mid m)=\operatorname{sn}(y, k)$, where $m=k^{2}$.
} 
where

$$
\Delta_{1}(t)=\left(\begin{array}{cc}
2 m x_{1}\left(1-x_{1}\right) \operatorname{sn} \frac{\partial z(t)}{\partial x_{1}}+\mathrm{dn}^{2} & 2 m x_{1}\left(1-x_{1}\right) \operatorname{sn} \frac{\partial z(t)}{\partial m}+x_{1}\left(1-x_{1}\right) \operatorname{sn}^{2} \\
\frac{\partial r(t)}{\partial x_{1}} & \frac{\partial r(t)}{\partial m}
\end{array}\right) .
$$

Let us denote

$$
\mathrm{sn}_{0}=z(0), \quad \mathrm{cn}_{0}=\mathrm{cn}\left(\psi_{0} \mid m\right), \quad \mathrm{dn}_{0}=\operatorname{dn}\left(\psi_{0} \mid m\right) .
$$

With a help of Mathematica we finally find the following expression:

$$
\operatorname{det} \Delta_{1}(t)=-\frac{M \mathrm{cnsndn}}{8}\left[M^{2} t^{2}-\frac{1}{1-m}\left(E_{M t}-\frac{\mathrm{dnsn}}{\mathrm{cn}}+\frac{\mathrm{dn}_{0} \mathrm{sn}_{0}}{\mathrm{cn}_{0}}\right)\left(E_{M t}+\frac{\mathrm{dncn}}{\mathrm{sn}}-\frac{\mathrm{dn}_{0} \mathrm{cn}_{0}}{\mathrm{sn}_{0}}\right)\right],
$$

where

$$
E_{M t}=\int_{\psi_{0}}^{M t+\psi_{0}} \operatorname{dn}^{2}(\xi \mid m) d \xi
$$

By construction, conjugate times $t_{*}$ are solutions of the equation $\operatorname{det} \Delta_{1}\left(t_{*}\right)=0$. It is not difficult to note that they are actually zeros of the expression in the square brackets of (12), i.e., solutions of the equation

$$
T^{2}-\frac{1}{1-m}\left(E_{T}-\frac{\mathrm{dn} \mathrm{sn}}{\mathrm{cn}}+\frac{\mathrm{dn}_{0} \mathrm{sn}_{0}}{\mathrm{cn}_{0}}\right)\left(E_{T}+\frac{\mathrm{dn} \mathrm{cn}}{\mathrm{sn}}-\frac{\mathrm{dn}_{0} \mathrm{cn}_{0}}{\mathrm{sn}_{0}}\right)=0,
$$

where $T=M t$.

Theorem 2. For all initial conditions $\psi_{0} \neq j K, j \in \mathbb{N}$, the first conjugate (to 0 ) time $t_{*}^{1}>\frac{2 K}{M}$.

This result is a direct consequence of the following lemmas.

Lemma 1. For any $0<\psi_{0}<K$, the smallest positive root $T_{*}^{1}$ of (13) is contained in the open interval $\left(2 K-\psi_{0}, 3 K-\psi_{0}\right)$, while if $K<\psi_{0}<2 K$ one has $T_{*}^{1} \in\left(K+\psi_{0}, 2 K+\psi_{0}\right)$.

Proof. Let us rewrite equation (13) in a more convenient form:

$$
(1-m) T^{2}=F(T)=F_{1}(T) F_{2}(T),
$$

where

$$
F_{1}(T)=\frac{\mathrm{dn} \mathrm{sn}}{\mathrm{cn}}-\frac{\mathrm{dn}_{0} \mathrm{sn}_{0}}{\mathrm{cn}_{0}}-E_{T}, \quad F_{2}(T)=-\frac{\mathrm{dn} \mathrm{cn}}{\mathrm{sn}}+\frac{\mathrm{dn}_{0} \mathrm{cn}_{0}}{\mathrm{sn}_{0}}-E_{T} .
$$

First, we observe that $F_{1}(0)=F_{2}(0)=0$ and

$$
F_{1}^{\prime}(T)=(1-m) \mathrm{sc}^{2}, \quad F_{2}^{\prime}(T)=\mathrm{cs}^{2},
$$

and, therefore, both $F_{1}$ and $F_{2}$ are monotone increasing functions ${ }^{2}$. The points $T=j K-\psi_{0}$, $j \in \mathbb{N}$, define their vertical asymptotes (with odd $j$ for $F_{1}$ and even $j$ for $F_{2}$ ). In addition, observe that $F_{1}(T) \rightarrow \pm \infty$ as $T \rightarrow(2 j-1) K-\psi_{0}$ from the left ( + sign) or from the right $(-$ sign), and $F_{1}(2 j K)<0$ for all $j \in \mathbb{N}$. At the same time, $F_{2}(T) \rightarrow \pm \infty$ as $T \rightarrow 2 j K-\psi_{0}$ as $T \rightarrow 2 j K-\psi_{0}$ from the left $(+$ sign $)$ or from the right $(-$ sign $)$. In addition, $F_{2}\left(K-\psi_{0}\right)>0$ and $F_{2}((2 j+1) K)<0$, for all $j=1,2, \ldots$.

Passing to the function $F=F_{1} F_{2}$, we see that $F(T)$ is positive on the first interval $\left(0, K-\psi_{0}\right)$ and tends to $+\infty$ as $T \rightarrow\left(K-\psi_{0}\right)$ from the left. Then, on $\left(K-\psi_{0}, 2 K-\psi_{0}\right), F(T)$ is strictly negative, while starting from the interval $\left(2 K-\psi_{0}, 3 K-\psi_{0}\right)$ it is monotone decreasing and goes to $+\infty$ or $-\infty$ as $T$ tends to the left or to the right terminal points of these intervals. In particular, $F(T)$ changes sign from negative to positive on each open interval of the form $\left(2 j K-\psi_{0},(2 j+1) K-\psi_{0}\right)$. This

\footnotetext{
${ }^{2}$ Here we use the standard notation $\mathrm{sc}=\frac{\mathrm{sn}}{\mathrm{cn}}, \mathrm{cs}=\frac{\mathrm{cn}}{\mathrm{sn}}$.
} 


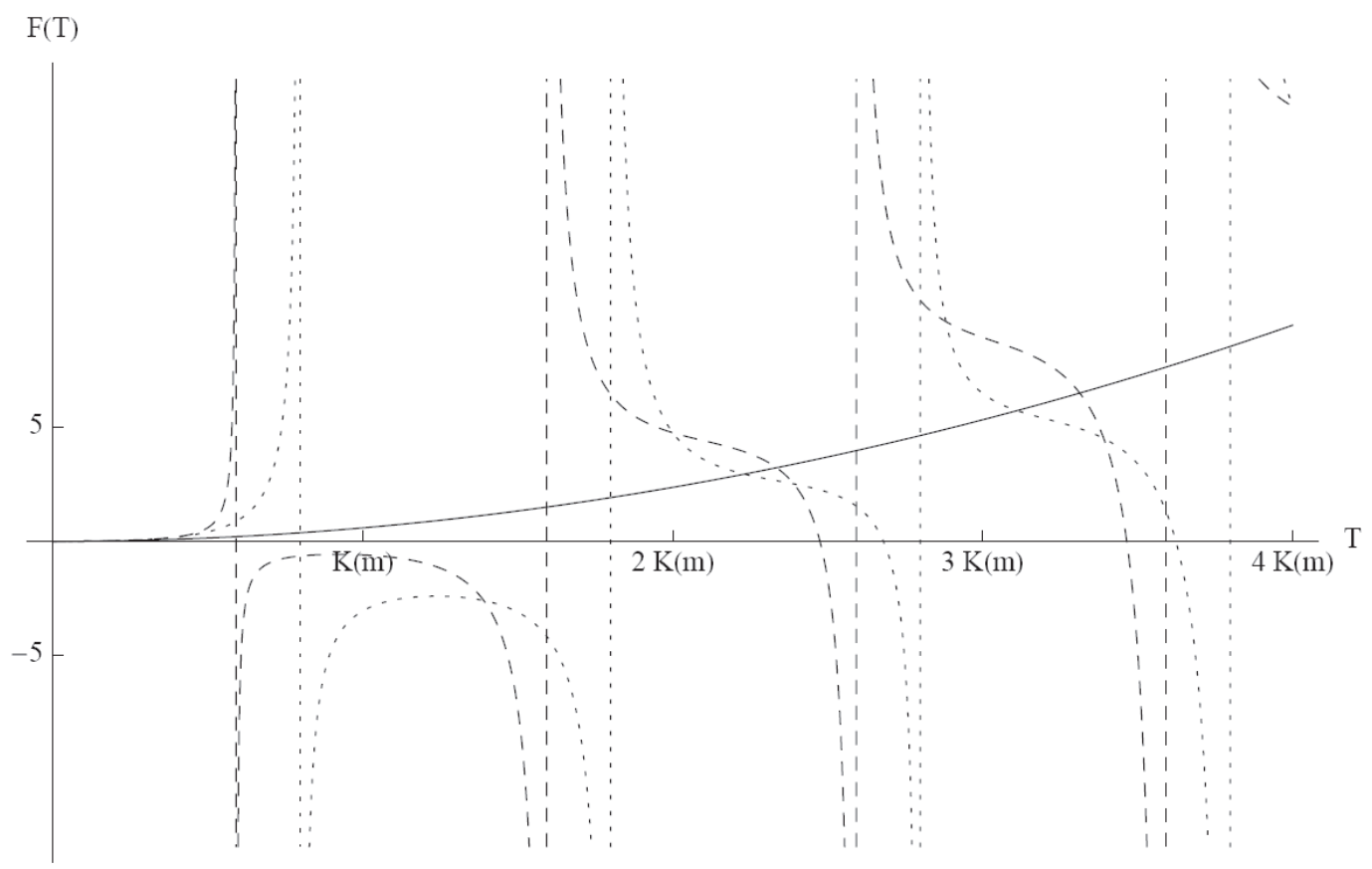

Fig. 5

implies that Eq. (13) has a solution on each open interval $\left(2 j K-\psi_{0},(2 j+1) K-\psi_{0}\right)$ starting from $j=1$.

To conclude, let us show that (13) has no solutions on the interval $\left(0, K-\psi_{0}\right.$ ] for $0<\psi_{0}<K$. First, observe that since the function $\operatorname{sc}^{2}(\xi \mid m)$ is monotone increasing from 0 to $+\infty$ on the interval $[0, K]$, for all $T \in\left[0, K-\psi_{0}\right]$ one has $F_{1}(T) \in\left[T(1-m) \mathrm{sc}_{0}^{2}, T(1-m) \mathrm{sc}^{2}\right]$ and $F_{2}(T) \in\left[T \mathrm{cs}^{2}, T \operatorname{cs}_{0}^{2}\right]$. Applying these estimates to the derivative of $F$ we get

$$
\frac{F^{\prime}(T)}{1-m}=\operatorname{sc}^{2} \int_{\psi_{0}}^{\psi_{0}+T} \operatorname{cs}^{2}(\xi \mid m) d \xi+\operatorname{cs}^{2} \int_{\psi_{0}}^{\psi_{0}+T} \operatorname{sc}^{2}(\xi \mid m) d \xi>T\left(1+\operatorname{sc}_{0}^{2} \operatorname{cs}^{2}\right)>0,
$$

and hence $F$ is monotone increasing on $\left[0, K-\psi_{0}\right]$ starting from the value $F(0)=0$. Further differentiation yields

$$
\frac{F^{\prime \prime}(T)}{1-m}=2+2 \operatorname{sn} \operatorname{cndn}\left(\frac{1}{\operatorname{cn}^{4}} \int_{\psi_{0}}^{\psi_{0}+T} \operatorname{cs}^{2}(\xi \mid m) d \xi-\frac{1}{\operatorname{sn}^{4}} \int_{\psi_{0}}^{\psi_{0}+T} \operatorname{sc}^{2}(\xi \mid m) d \xi\right) \geq 2,
$$

the equality taking place at $T=0$. So, for all $T \in\left(0, K-\psi_{0}\right]$ the function $F$ is strictly convex, and its graph lies above the parabola $(1-m) T^{2}$, which means that $(13)$ has no roots on this interval. Hence the smallest positive root of the equation $(1-m) T^{2}=F(T)$ is contained in $\left(2 K-\psi_{0}, 3 K-\psi_{0}\right)$.

Repeating the same arguments in the case $\psi_{0}=K+\varepsilon, 0<\varepsilon<K$, we easily show that

$$
T_{*}^{1} \in(2 K-\varepsilon, 3 K-\varepsilon)=\left(K+\psi_{0}, 2 K+\psi_{0}\right) .
$$

The lemma is proved.

The estimates of Lemma 1 do not allow one to say whether the first conjugate point occur before or after the period $\frac{2 K}{M}$ of the $\varphi$ variable. To answer to this question we use the following fact. 


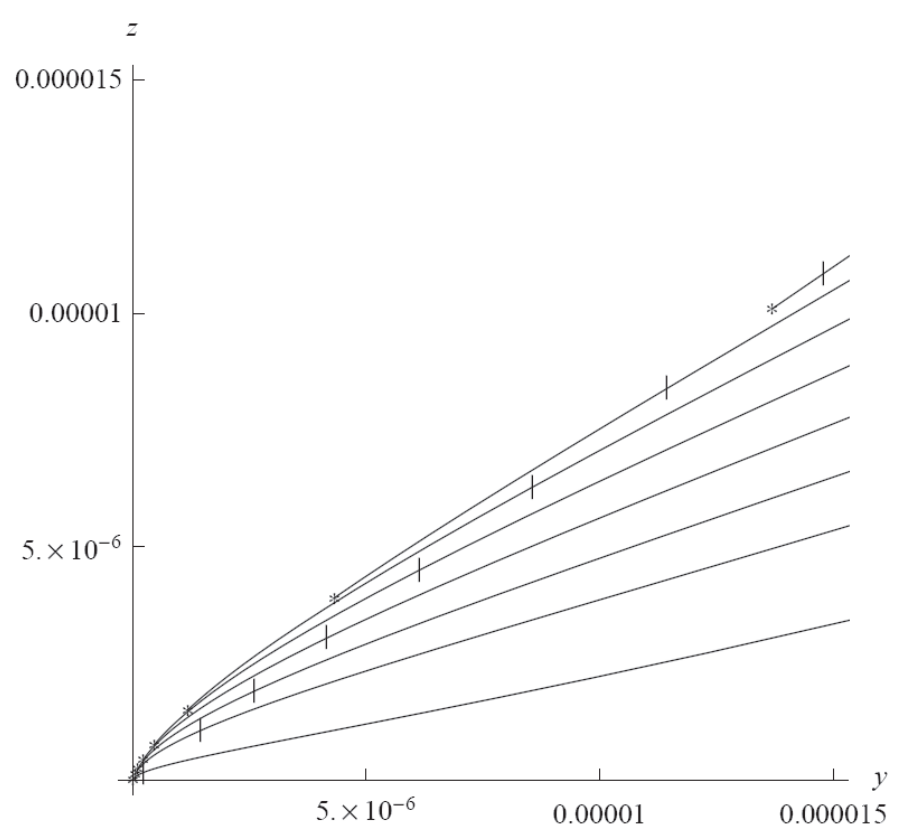

Fig. 6

Lemma 2. For all $\psi_{0} \neq j K, j \in \mathbb{N}, F(2 K)=E(2 K)^{2}$, where $E$ denotes a complete elliptic integral of second kind: $E(2 K)=E(\operatorname{am}(2 K \mid m) \mid m)$.

Proof. Observe that

$$
E_{2 K}=\int_{\psi_{0}}^{\psi_{0}+2 K} \operatorname{dn}(s \mid m)^{2} d s=E(2 K) .
$$

Then the symmetry of the elliptic functions with respect to half-periods yields

$$
\begin{aligned}
& F_{1}(2 K)=\frac{\operatorname{dn}\left(2 K+\psi_{0} \mid m\right) \operatorname{sn}\left(2 K+\psi_{0} \mid m\right)}{\operatorname{cn}\left(2 K+\psi_{0} \mid m\right)}-\frac{\operatorname{dn}\left(\psi_{0} \mid m\right) \operatorname{sn}\left(\psi_{0} \mid m\right)}{\operatorname{cn}_{0}\left(\psi_{0} \mid m\right)}-E_{2 K}=-E_{2 K}, \\
& F_{2}(2 K)=-\frac{\operatorname{dn}\left(2 K+\psi_{0} \mid m\right) \operatorname{cn}\left(2 K+\psi_{0} \mid m\right)}{\operatorname{sn}\left(2 K+\psi_{0} \mid m\right)}+\frac{\operatorname{dn}\left(\psi_{0} \mid m\right) \operatorname{cn}\left(\psi_{0} \mid m\right)}{\operatorname{sn}_{0}\left(\psi_{0} \mid m\right)}-E_{2 K}=-E_{2 K},
\end{aligned}
$$

and the result follows.

Lemma 3. If $\psi_{0} \neq j K, j \in \mathbb{N}$, then $T_{*}^{1}>2 K$.

Proof. Let us prove the result for $0<\psi_{0}<K$, the case $K<\psi_{0}<2 K$ being completely similar. By Lemma $1, T_{*}^{1} \in\left(2 K-\psi_{0}, 3 K-\psi_{0}\right)$, and at the same time $2 K \in\left(2 K-\psi_{0}, 3 K-\psi_{0}\right)$. In addition, by Lemma 2, $F(2 K)=E(2 K)^{2}$, while $F\left(T_{*}^{1}\right)=4(1-m) K^{2}(m)$. Taking into account that $F$ is monotone decreasing on $\left(2 K-\psi_{0}, 3 K-\psi_{0}\right)$, and $E(2 K)^{2} \geq 4(1-m) K^{2}(m)$ for all $m \in(0,1)$, we conclude that $T_{*}>2 K$.

Figure 5 shows the possible disposition of the graph of $F(T)$ for $\psi_{0} \in(0, K)$ (dashed line) and $\psi_{0} \in(K, 2 K)$ (dotted line) with respect to the parabola $(1-m) T^{2}$.

To complete our analysis, consider now the case $\dot{\varphi}(0)=0$, i.e., $\psi_{0}=0 \bmod K$.

Theorem 3. For $\psi_{0}=j K, j=0,1, \ldots$, the first conjugate (to 0 ) time is $t_{*}^{1}=2 K / M$. 
Proof. First, for all initial points we have

$$
\dot{\varphi}(0)=\frac{\dot{x}(0)}{2 \sin \varphi_{0} \cos \varphi_{0}}=\frac{x_{1} x_{2}\left(x_{2}-x_{1}\right) \mathrm{sn}_{0} \mathrm{cn}_{0} \mathrm{dn}_{0}}{\sin \varphi_{0} \cos \varphi_{0}\left(x_{2}-\left(x_{2}-x_{1}\right) \mathrm{sn}_{0}^{2}\right)^{2}} .
$$

Substituting this expression in (11) we obtain

$$
\operatorname{det} D_{\left(p_{\varphi}(0), p_{r}\right)} \Phi=\frac{4 x_{1}^{2} \operatorname{sn}_{0} \mathrm{cn}_{0} \mathrm{dn}_{0}}{\delta^{3} \sin \varphi_{0} \cos \varphi_{0}\left(x_{2}-\left(x_{2}-x_{1}\right) \mathrm{sn}_{0}^{2}\right)^{2}} .
$$

On the other hand, according to our previous calculations,

$$
\operatorname{det} D_{\left(x_{1}, m\right)} G=\frac{x_{2}^{2} \operatorname{det} \Delta_{1}(t)}{2 \sin \varphi \cos \varphi\left(x_{2}-\left(x_{2}-x_{1}\right) \operatorname{sn}_{0}^{2}\right)^{2}}
$$

where $\operatorname{det} \Delta_{1}$ is given by (12).

Consider first the case $\psi_{0}=0$. Then $\mathrm{sn}_{0}=0$, while $\mathrm{cn}_{0} \mathrm{dn}_{0}=1$. In particular,

$$
\left.\operatorname{sn}_{0} \operatorname{det} \Delta_{1}\right|_{\mathrm{sn}_{0}=0}=-\frac{M \mathrm{cnsndndn} \mathrm{dn}_{0} \mathrm{cn}_{0}}{8(1-m)}\left(E_{M t}-\frac{\mathrm{dn} \mathrm{sn}}{\mathrm{cn}}\right) .
$$

Therefore, since by construction $\exp _{\left(\varphi_{0}, r_{0}\right), t}=G \circ \Phi$, the endpoint mapping is not an immersion if and only if

which is equivalent to the equation

$$
\operatorname{det} D_{\left(p_{\varphi}(0), p_{r}\right)} \Phi \operatorname{det} D_{\left(x_{1}, m\right)} G=0,
$$

$$
\operatorname{cnsndn}\left(E_{M t}-\frac{\mathrm{dn} \mathrm{sn}}{\mathrm{cn}}\right)=0 .
$$

It is easy to see that, because of the singularity in the bracket term, the points $\frac{(2 j+1) K}{M}$ are not solutions to (14). Adopting the analysis of the function $F_{1}$ introduced in the proof of Lemma 1 to the case $\psi_{0}=0, K, \ldots$, we see that the first zero of the bracket term of (14) is contained in the interval $\left(\frac{2 K}{M}, \frac{3 K}{M}\right)$, and is strictly greater than $\frac{2 K}{M}$. Hence the smallest root of this equations comes from the condition $\operatorname{sn}(M t \mid m)=0$, and thus $t_{*}^{1}=\frac{2 K}{M}$.

The same argument with a few obvious modifications remains true for $\psi_{0}=K$, and the theorem follows.

In Fig. 6 we present an example of a family of orbits, starting at $r_{0}=-1$ and $x_{0}=0.35$ for $\delta=3, p_{r}=0.01$, and $\gamma_{+}=6.1$, calculated up to the first conjugate point (marked by "**"). The polar coordinates of the initial point in this example are $\rho_{0}=e^{-1}$ and $\varphi_{0} \approx 0.633052$. The short periodic solutions for $\varphi$ exist in the interval $\bar{h} \in(-1.11,0)$. The solutions on the figure correspond to $\bar{h}=-1,-0.9,-0.8,-0.7,-0.6,-0.5,-0.4$ and -0.2 . The sign "" marks the end of the first period of $\varphi(t)$. The illustrated solutions asymptotically tend to the origin but lose the optimality before, just after the end of the first period.

\section{Numerical Computation of Cut and Conjugate Loci}

In order to compute the conjugate and cut loci of the energy minimization problem we consider the level sets of the Hamiltonian $H_{n}$

$$
\frac{1}{2}\left(p_{\varphi}+\delta \cos \varphi \sin \varphi\right)^{2}+p_{r}\left(\delta \sin ^{2} \varphi-\gamma_{+}\right)=h+\delta^{2} \cos \varphi^{2} \sin \varphi^{2},
$$

with $\delta$ and $\gamma_{+}$fixed. Given an initial point $\varphi_{0}$, for each pair $p_{r}$ and $\bar{h}=h+p_{r} \gamma_{+}$there are two normal extremals corresponding to

$$
p_{\varphi}(0)= \pm \sqrt{2\left(\bar{h}-W\left(\sin ^{2} \varphi_{0}\right)\right)}-\delta \cos \varphi_{0} \sin \varphi_{0} .
$$


Varying $p_{r}$ and $h$, we obtain two-parametric families of normal extremals issuing from $\varphi_{0}$.

In order to compute the conjugate locus we used the following method. First we fix the intervals of variation of $p_{r}$ and $h$. The discretization of these intervals gives a net of points $\left(p_{r}^{i}, h^{j}\right), i, j=1, \ldots, N$, where $N$ should be taken large enough. Taking both positive and negative branches of $\dot{\varphi}$, we obtain at most $2 N^{2}$ extremals starting from $\varphi_{0}$. Using the Hampath code [8], on this set of extremals we compute numerically the conjugate points occurring at a fixed final time $t_{f}$, and the values of the cost functional. As the result, we obtain the section $t=t_{f}$ of the conjugate locus, as well as the isocost curves. It is worth nothing that the terminal time $t_{f}$ is an important parameter of the problem. Since the energy level set in the meridian case is not compact, in order to obtain a meaningful picture, the value of $t_{f}$ should be chosen in accordance with the intervals of variation of $p_{r}$ and $h$. In our tests we chose $t_{f}$ close to the average value of the first conjugate times predicted in Sec. 5 .

The results displayed in Figs. 7 and 8 give an example of the optimal synthesis in the 2D integrable case for $\delta=5, \gamma_{+}=10.1, t_{f}=1.7$, and $\varphi_{0}=\arcsin 2 / \sqrt{5}$. Thick curves represent the components of the conjugate locus corresponding to short $(S)$ and long $\left(L_{1}\right.$ and $\left.L_{2}\right)$ orbits. Other points indicate the isocost lines, while thick black curves represent the cut locus. In our tests no conjugate points were detected along the trajectories corresponding to rotations.

As we saw in Sec. 4, the type of dynamics of the problem is stipulated by the behavior of the function $W$, which is completely determined by the values of $\delta$ and $p_{r}$. For a fixed value of $\delta$ (in our example $\delta=5)$, the variation of $p_{r} \in[-\alpha, \alpha]$ for $\alpha>0$ large enough results in the transformation of the graph of the potential $W$ from the left to the right column in Fig. 3, passing through the configurations indicated on Fig. 2. In particular, for the negative values of $p_{r}$ on different level sets of $h$ we obtain trajectories with $\varphi(t) \in\left[-\frac{\pi}{2}, 0\right]$, while if $p_{r}>0 \varphi(t) \in[0, \pi]$ for $t \in\left[0, t_{f}\right]$. As a consequence, the part of the conjugate locus corresponding to long trajectories consists of two distinct branches, indicated as $L_{1}$ and $L_{2}$ on Fig. 7a. If we enlarge the intervals of the variation of $p_{r}$ and $h$, these two branches cross each other, as we see in Fig. 7b. We leave for the future the detailed analysis of the appearing singularity. The component of the cut locus related to the long extremals consists of two branches corresponding to $\varphi_{c}^{1}=\pi-\varphi_{0}$ and $\varphi_{c}^{2}=-\varphi_{0}$. It is formed by the intersections of the long trajectories lying on the same levels of $h$ and $p_{r}$, as described in Proposition 6. The conjugate locus has cusps at $\varphi_{c}^{1}$ (the $L_{1}$ part) and $\varphi_{c}^{2}$ (the $L_{2}$ part).

The part of the conjugate locus formed by the conjugate points along short orbits is a closed curve with two cusps at $\varphi_{0}$. These points are the extremities of the cut locus $\varphi_{c}^{3}=\varphi_{0}$, which again is formed by the intersections of the pairs of trajectories lying on the same levels of $h$ and $p_{r}$ and issuing from $\varphi_{0}$ with $\pm \dot{\varphi}(0)$, as described in Proposition 5. Figure 8 illustrates in detail this part of the locus. The isocost lines are indicated by gray points. The self-intersections of the isocost curves form the cut locus as, for example, the isocost curve shown in Fig. 8.

Let us note that the structure of the cut locus corresponding to short orbits is different from its analog in the spherical case described in [6]: in the spherical case cut points occur on different level sets of $h$. In Fig. 9 we present an example of the computation of the cut and conjugate loci in the spherical case. The similarity in the form of the $L_{1}$ part of the conjugate locus with the conjugate locus in the spherical case comes from the similar dynamics of $r$ and $\theta$ variables, as it can be seen from our parametrization formulas. In contrast, it the difference in the cut loci for short orbits is remarkable. 


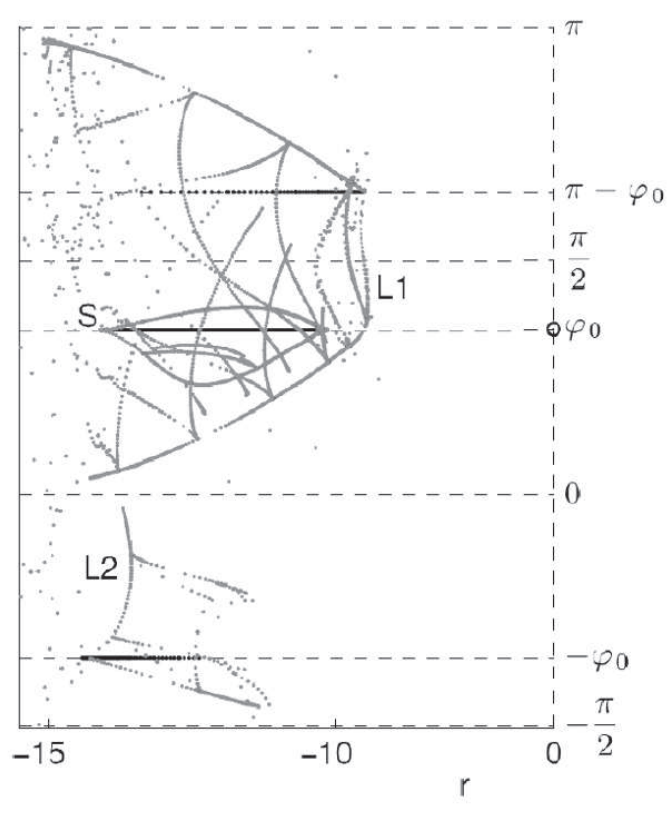

a

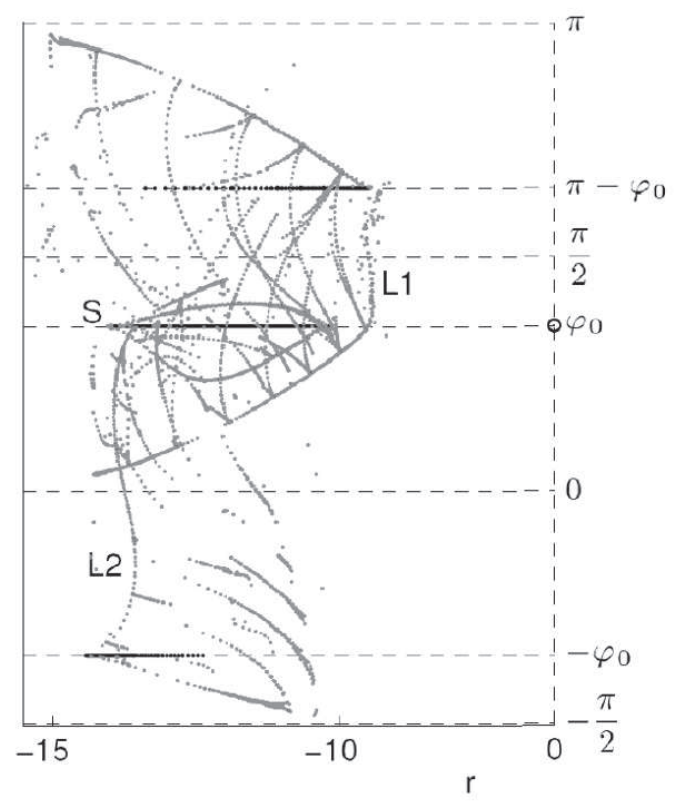

$\mathrm{b}$

Fig. 7. Conjugate and cut loci in the $2 \mathrm{D}$ case. Case a: $\bar{h} \in[-14.9,5], p_{r} \in[-3,1]$. Case b: $\bar{h} \in[-14.9,15], p_{r} \in[-3,3]$.

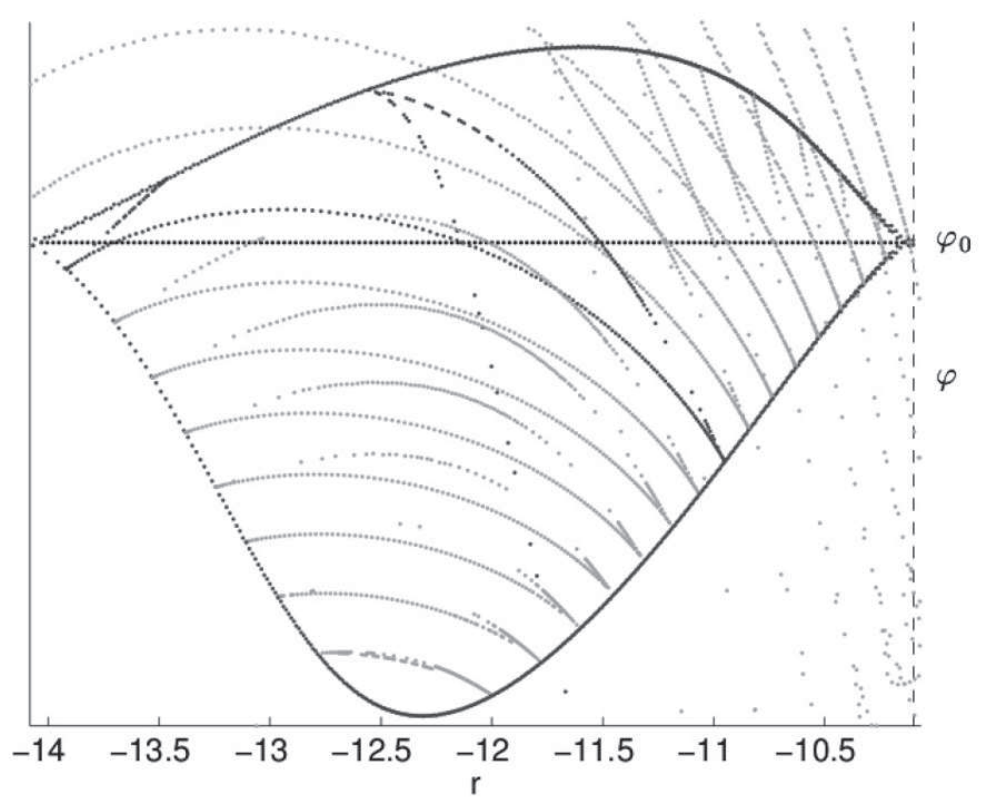

Fig. 8. Conjugate and cut loci associated to short orbits from the previous figure.

\section{Nonintegrable Case $\gamma_{-} \neq 0, p_{\theta}=0$}

In this section, we discuss the effect of the dissipation parameter $\gamma_{-} \neq 0$ on the set of extremals. Recall that the $2 \mathrm{D}$-system is given in Cartesian coordinates by the equations

$$
\begin{aligned}
& \dot{y}=-\Gamma y-u z, \\
& \dot{z}=\gamma_{-}-\gamma_{+} z+u y,
\end{aligned}
$$




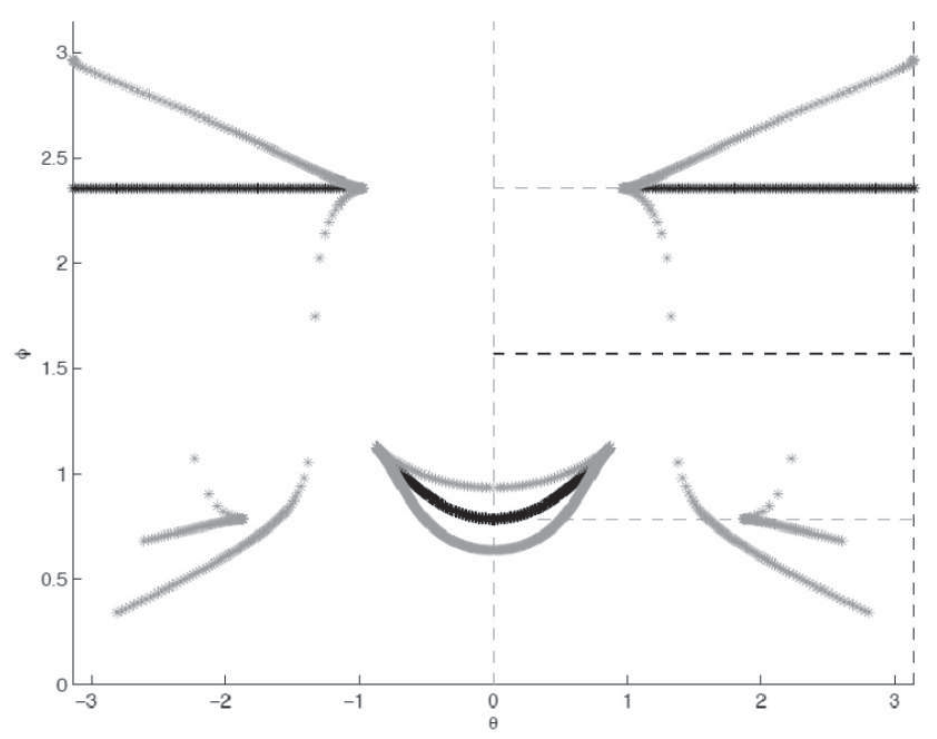

Fig. 9. Example of conjugate and cut loci in the spherical integrable case $p_{\theta} \neq 0$, $p_{r}=0$. The smile-shaped contour in the center is the conjugate locus of short orbits.

and the singular trajectories are given by $y=0$ and $z=z_{0}=\frac{\gamma_{-}}{2\left(\gamma_{+}-\Gamma\right)}$. We use the polar coordinates $y=\rho \sin \varphi, z=\rho \sin \varphi$ in order to normalize the control directly. Indeed, it is not possible to normalize simultaneously the singular lines and the control direction if $\gamma_{-} \neq 0$. By setting $r=\ln \rho, \delta=\gamma_{+}-\Gamma$, the system takes the form

$$
\begin{aligned}
& \dot{r}=-\Gamma-\delta \cos ^{2} \varphi+\gamma_{-} \cos \varphi e^{-r}, \\
& \dot{\varphi}=-\gamma_{-} \sin \varphi e^{-r}+\frac{\delta \sin (2 \varphi)}{2}-u .
\end{aligned}
$$

The normal Hamiltonian is given by

$$
H_{n}=p_{r}\left(\gamma_{-} \cos \varphi e^{-r}-\left(\Gamma+\delta \cos ^{2} \varphi\right)\right)+p_{\varphi}\left(-\gamma_{-} \sin \varphi e^{-r}+\frac{\delta \sin (2 \varphi)}{2}\right)+\frac{p_{\varphi}^{2}}{2} .
$$

Again, since $\dot{\varphi}=p_{\varphi}+\delta \sin \varphi \cos \varphi-\gamma_{-} \sin \varphi e^{-r}$, the equation $H_{n}=h$ can be written as follows:

$$
\frac{\dot{\varphi}^{2}}{2}+V\left(\varphi, p_{r}, r\right)=h,
$$

where

$$
V\left(\varphi, p_{r}, r\right)=p_{r}\left(\gamma_{-} \cos \varphi e^{-r}-\left(\Gamma+\delta \cos ^{2} \varphi\right)\right)-\frac{1}{2}\left(\frac{\delta \sin (2 \varphi)}{2}-\gamma_{-} \sin \varphi e^{-r}\right)^{2} .
$$

Observe that in contrast to the integrable case, if $\gamma_{-} \neq 0$ we have a coupling between the evolution of $\varphi$ and the $r$, which can be interpreted as a dissipation effect.

To simplify the further analysis, we pass again to Cartesian coordinates. Then

$$
H_{n}=-\Gamma y p_{y}+p_{z}\left(\gamma_{-}-\gamma_{+} z\right)+\frac{1}{2}\left(y p_{z}-z p_{y}\right)^{2} .
$$

Denote

$$
P=y p_{z}-z p_{y}, \quad Q=y p_{y}+z p_{z} .
$$


Then we obtain

$$
\begin{aligned}
\dot{y} & =-\Gamma y-z P, \\
\dot{z} & =\left(\gamma_{-}-\gamma_{+} z\right)+y P, \\
\dot{p}_{y} & =\Gamma p_{y}-p_{z} P, \\
\dot{p}_{z} & =\gamma_{+} p_{z}+p_{y} P,
\end{aligned}
$$

which yields

$$
\begin{aligned}
& \dot{Q}=\gamma_{-} p_{z}, \\
& \dot{P}=-\delta\left(y p_{z}+z p_{y}\right)-\gamma_{-} p_{y} .
\end{aligned}
$$

The quantities $P$ and $Q$ correspond to dual polar coordinates on fibers, which are well defined provided $y^{2}+z^{2} \neq 0$. The equilibrium points can be easily computed. If $\gamma_{-} \neq 0$, one has $p_{z}=0$. Hence $\dot{p}_{y}=0$ gives $p_{y}=0$ if $\Gamma \neq 0$. In addition, $\dot{y}=0$ yields $y=0$ and $\dot{z}=0$ implies $z=\gamma_{-} / \gamma_{+}$. This corresponds to the equilibrium point of the free motion. Additional critical points can occur at infinity. Indeed, due to the dissipation, the Poisson-stable point does not exist and from the Hopf theorem almost every point is departing [12]. Since the state variables remain bounded, we deduce that the adjoint vector $|p| \rightarrow \infty$ as $t \rightarrow \infty$. To analyze this behavior, one can use the Poincaré compactification

$$
\begin{aligned}
\dot{y} & =-\Gamma y w^{2}+z\left(z p_{y}-y p_{z}\right), \\
\dot{z} & =\gamma_{-} w^{3}-\gamma_{+} w^{2}+y\left(y p_{z}-z p_{y}\right), \\
\dot{p}_{y} & =\Gamma p_{y} w^{2}+p_{z}\left(z p_{y}-y p_{z}\right), \\
\dot{p}_{z} & =\gamma_{+} p_{z} w^{2}+\left(y p_{z}-z p_{y}\right) p_{y}, \\
\dot{w} & =1 .
\end{aligned}
$$

Another way to describe this phenomenon is to parametrize the adjoint variables as follows:

$$
p_{y}=\varrho \cos \vartheta, \quad p_{z}=\varrho \sin \vartheta,
$$

where $(\varrho, \vartheta)$ define a coordinate system on fibers if $\varrho \neq 0$, and

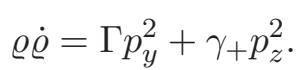

Then we have

$$
\Gamma p_{y}^{2}+\gamma_{+} p_{z}^{2} \geq \gamma_{+} \varrho^{2}
$$

since $2 \Gamma \geq \gamma_{+} \geq 0$. In particular, $\varrho(t) \geq e^{\gamma_{+} t} \varrho(0)$, and hence $|p| \rightarrow+\infty$ if $t \rightarrow+\infty$, provided $\varrho(0) \neq 0$. For the new variables we obtain

$$
\begin{aligned}
& \dot{\varrho}=\varrho\left(\Gamma+\delta \sin ^{2} \vartheta\right), \\
& \dot{\vartheta}=\delta \sin (2 \vartheta) / 2+P, \\
& \dot{P}=\delta Q-\gamma_{-} \varrho \cos \vartheta, \\
& \dot{Q}=\gamma_{-} \varrho \sin \vartheta .
\end{aligned}
$$

In this representation $P$ is the control. Numerical simulations can be used to analyze the limit behaviors of $P$ and $Q$. They are represented in Fig. 10 . 

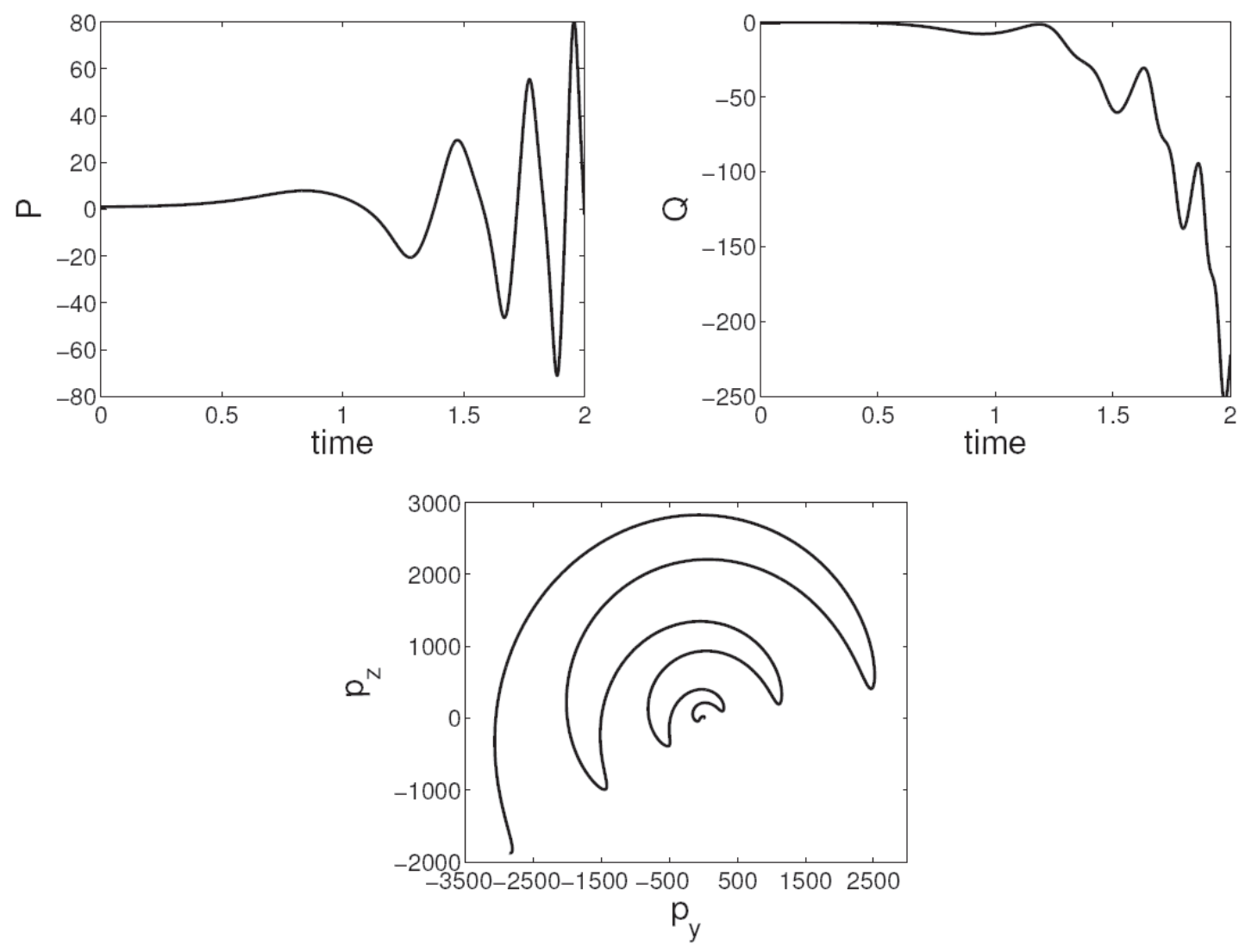

Fig. 10. Evolution of the reduced coordinates $P$ (top), $Q$ (middle) and $\left(p_{y}, p_{z}\right)$ (bottom). Numerical values are taken to be $\Gamma=5, \gamma_{+}=2, \gamma_{-}=-1, \rho(0)=1$, and $\theta(0)=0$.

\section{Summary of the Results and Possible Extensions}

Above we presented a detailed analysis of the flat 2D optimal solutions of the energy minimization problem for a two-level dissipative quantum control system described by (1). It is a control system of affine type and the behavior of its extremals is very intricate, but the integrability of the problem in the case $\gamma_{-}=0$ allows one to compute explicitly the exponential mapping in terms of the Jacobi elliptic functions. In the present paper we use this fact in order to obtain an explicit characterization of the cut and conjugate loci of the problem. At the end of a quite nontrivial computation, we found an implicit equation describing the conjugate points along one specific class of the normal extremals, namely, for the short periodic orbits, and obtain sharp estimates for the corresponding first conjugate times. Due to the high complexity of the problem, in order to construct the optimal synthesis of the $2 \mathrm{D}$ case we then use numerical simulations. Finally, we also provided a first insight into the asymptotic behavior of the flat optimal solutions in the general nonintegrable case.

In our further study we plan to extend our methodology in order to characterize all classes of the solutions of the 2D flat integrable problem. We also plan to adopt our method of computation to the spherical case and to obtain analytical estimates for the conjugate times in this case. This would be an important step toward understanding the structure of the optimal trajectories of the general 3D problem. 


\section{Appendix}

Here we list the explicit formulas for $x(t)=\sin ^{2} \varphi(t), \theta(t)$ and $r(t)$ along the normal trajectories of the energy minimization problem for (1) in the integrable case $\gamma_{-}=0$. They are obtained via direct integration of the natural mechanical system (7) and the additional equations (8) as described in Sec. 4. According to the different values of the constants of motion $p_{\theta}, p_{r}$ and $h$, and of the dissipation parameters $\Gamma$ and $\delta$, we have to distinguish between 3D trajectories and 2D trajectories lying in the meridian planes of the Bloch ball. The corresponding dynamics of the $\varphi$-variable is illustrated in Figs. 1-3.

I. 3D case: $\gamma_{-}=0, p_{\theta} \neq 0$.

Ia. Case $\delta^{2}>p_{\theta}^{2}-2 \delta p_{r}$ (Fig. 1, left).

* According to our phase portraits classification, there are three parallel orbits corresponding to $\varphi_{*}^{1}=\arcsin \sqrt{x_{*}}, \varphi_{*}^{2}=\pi-\arcsin \sqrt{x_{*}}$, and $\varphi_{*}^{0}=\frac{\pi}{2}, x_{*}$ being the unique positive real root of the equation $W^{\prime}\left(x_{*}\right)=0$. The first two of this orbits lie on the level sets $\bar{h}=W\left(x_{*}\right)$, and the third one on $\bar{h}=W(1)=\frac{p_{\theta}^{2}}{2}+\delta p_{r}$.

* On each level set $\bar{h} \in\left(W\left(x_{*}\right), W(1)\right)$ there are two families of small periodic solutions, symmetrically placed with respect to the equatorial plane and centered at $\varphi_{*}^{1,2}$, respectively. We have $0<x_{1}<x_{2}<1$,

$$
\begin{gathered}
k^{2}=\frac{\left(1-x_{3}\right)\left(x_{2}-x_{1}\right)}{\left(x_{2}-x_{3}\right)\left(1-x_{1}\right)}, \quad M=\sqrt{\delta^{2}\left(1-x_{1}\right)\left(x_{2}-x_{3}\right)}, \\
x(t)=\frac{-x_{1}\left(x_{2}-x_{3}\right)+x_{3}\left(x_{2}-x_{1}\right) z^{2}(t)}{-\left(x_{2}-x_{3}\right)+\left(x_{2}-x_{1}\right) z^{2}(t)}, \quad \varphi(t)=\arcsin \sqrt{x(t)},
\end{gathered}
$$

while the remaining variables are given by

$$
\begin{aligned}
\theta(t)-\theta(0)= & \frac{\left(1-x_{3}\right) p_{\theta} t}{x_{3}}+\frac{p_{\theta}\left(x_{3}-x_{1}\right)}{x_{1} x_{3} M}\left(\Pi\left[\frac{x_{3}\left(x_{2}-x_{1}\right)}{x_{1}\left(x_{2}-x_{3}\right)}, \operatorname{am}\left(M t+\psi_{0}, k\right), k\right]-\right. \\
& \left.-\Pi\left[\frac{x_{3}\left(x_{2}-x_{1}\right)}{x_{1}\left(x_{2}-x_{3}\right)}, \operatorname{am}\left(\psi_{0}, k\right), k\right]\right), \\
r(t)-r(0)= & \left(\delta x_{3}-\gamma_{+}\right) t+\frac{\delta\left(x_{1}-x_{3}\right)}{M}\left(\Pi\left[\frac{x_{2}-x_{1}}{x_{2}-x_{3}}, \operatorname{am}\left(M t+\psi_{0}, k\right), k\right]-\right. \\
& \left.-\Pi\left[\frac{x_{2}-x_{1}}{x_{2}-x_{3}}, \operatorname{am}\left(\psi_{0}, k\right), k\right]\right) ;
\end{aligned}
$$

* each level set $\bar{h}>W(1)$ contains a unique long periodic trajectory crossing the equatorial plane. It is characterized by $0<x_{1}<1<x_{2}$ and thus admits the following parametrization:

$$
\begin{gathered}
k^{2}=\frac{\left(x_{2}-x_{3}\right)\left(1-x_{1}\right)}{\left(1-x_{3}\right)\left(x_{2}-x_{1}\right)}, \quad M=\sqrt{\delta^{2}\left(x_{2}-x_{1}\right)\left(1-x_{3}\right)}, \\
x(t)=\frac{-x_{1}\left(1-x_{3}\right)+x_{3}\left(1-x_{1}\right) z^{2}(t)}{-\left(1-x_{3}\right)+\left(1-x_{1}\right) z^{2}(t)} .
\end{gathered}
$$


Since these orbits cross the equatorial plane, $\varphi(t)$ has to be computed using either arcsin or $\pi-\arcsin$ in order to obtain an analytic function. For $\theta$ and $r$ we obtain

$$
\begin{aligned}
\theta(t)-\theta(0)= & \frac{\left(1-x_{3}\right) p_{\theta} t}{x_{3}}+\frac{p_{\theta}\left(x_{3}-x_{1}\right)}{x_{1} x_{3} M}\left(\Pi\left[\frac{x_{3}\left(1-x_{1}\right)}{x_{1}\left(1-x_{3}\right)}, \operatorname{am}\left(M t+\psi_{0}, k\right), k\right]-\right. \\
& \left.-\Pi\left[\frac{x_{3}\left(1-x_{1}\right)}{x_{1}\left(1-x_{3}\right)}, \operatorname{am}\left(\psi_{0}, k\right), k\right]\right), \\
r(t)-r(0)= & \left(\delta x_{3}-\gamma_{+}\right) t-\frac{b\left(x_{1}-x_{3}\right)}{M}\left(\Pi\left[\frac{1-x_{1}}{1-x_{3}}, \operatorname{am}\left(M t+\psi_{0}, k\right), k\right]-\right. \\
& \left.-\Pi\left[\frac{1-x_{1}}{1-x_{3}}, \operatorname{am}\left(\psi_{0}, k\right), k\right]\right) ;
\end{aligned}
$$

* the level set $\bar{h}=W(1)$ also carries on separatrices, which can be computed as limit cases for the two families of periodic orbits since $\lim _{k \rightarrow 1} \operatorname{sn}(u, k)=\tanh u$.

Ib. Case $\delta^{2} \leq p_{\theta}^{2}-2 \delta p_{r}$ (Fig. 1, right). There only two types of solutions: parallel orbits $\varphi=\pi / 2$ lying in the equatorial plane, and long periodic trajectories. Their parametrization is the same as in the case Ia.

II. Meridian 2D case: $\gamma_{-}=0, p_{\theta}=0$. As we have shown above, new classes of motion can appear. In addition, we have $x_{3}=0$ and

$$
x_{1}=a-\sqrt{a^{2}+\frac{2 \bar{h}}{\delta^{2}}}, \quad x_{2}=a+\sqrt{a^{2}+\frac{2 \bar{h}}{\delta^{2}}} .
$$

IIa. $0<\left|p_{r}\right|<\frac{|\delta|}{2}$ (Fig. 2).

* Parallel solutions: For all values of $p_{r}$ on the level sets $\bar{h}=\delta p_{r}$ and $\bar{h}=0$ we have parallel solutions $\varphi=0$ and $\varphi=\pi / 2$, which in the meridian case results in horizontal and vertical axes on the $y z$-plane. In addition, on the level set $\bar{h}=-\delta^{2} a^{2} / 2$ we have a couple of solutions characterized by $\varphi= \pm \arcsin \sqrt{a} \bmod \pi$, which define a straight line in each quadrant of the $y z$-plane. These lines correspond to $\pi / 4$ if $p r=-\delta / 2$;

* Short orbits $-\delta^{2} a^{2} 2<\bar{h}<\delta p_{r}<0$ or $-\delta^{2} a^{2} / 2<\bar{h}<0<\delta p_{r}$, and $0<x_{1}<x_{2}<1$. Then

$$
\begin{aligned}
x(t) & =\frac{x_{1} x_{2}}{x_{2}-\left(x_{2}-x_{1}\right) \operatorname{sn}^{2}\left(M t+\psi_{0}, k\right)}, \\
r(t)-r(0) & =\frac{\delta x_{1}}{M}\left(\Pi\left(\frac{x_{2}-x_{1}}{x_{2}}, \operatorname{am}\left(M t+\psi_{0}, k\right), k\right)-\Pi\left(\frac{x_{2}-x_{1}}{x_{2}}, \operatorname{am}\left(\psi_{0}, k\right), k\right)\right)-\gamma_{+} t
\end{aligned}
$$

where

$$
M^{2}=\delta^{2} x_{2}\left(1-x_{1}\right), \quad k^{2}=\frac{x_{2}-x_{1}}{x_{2}\left(1-x_{1}\right)} .
$$

The period of this class of orbits is $\frac{2 K(k)}{M}$.

$*$ Long orbits $\delta p_{r}<\bar{h}<0$ and $0<x_{1}<1<x_{2}$. Then

$$
\begin{aligned}
x(t) & =\frac{x_{1}}{1-\left(1-x_{1}\right) \operatorname{sn}^{2}\left(M t+\psi_{0}, k\right)}, \\
r(t)-r(0) & =\frac{\delta x_{1}}{M}\left(\Pi\left(1-x_{1}, \operatorname{am}\left(M t+\psi_{0}, k\right), k\right)-\Pi\left(1-x_{1}, \operatorname{am}\left(\psi_{0}, k\right), k\right)\right)-\gamma_{+} t,
\end{aligned}
$$

where

$$
M^{2}=\delta^{2}\left(x_{2}-x_{1}\right), \quad k^{2}=\frac{x_{2}\left(1-x_{1}\right)}{x_{2}-x_{1}} .
$$

These orbits are periodic with the period $\frac{4 K(k)}{M}$. 
* Long orbits $0<\bar{h}<\delta p_{r}$. This case is analog to the previous one up to shifting along the $\varphi$-axis. More precisely, we have $x_{1}<0<x_{2}<1$, and

$$
\begin{aligned}
x(t) & =1-\frac{1-x_{2}}{1-x_{2} \operatorname{sn}^{2}\left(M t+\psi_{0}, k\right)}, \\
r(t)-r(0) & =-\frac{\delta\left(1-x_{2}\right)}{M}\left(\Pi\left(x_{2}, \operatorname{am}\left(M t+\psi_{0}, k\right), k\right)-\Pi\left(x_{2}, \operatorname{am}\left(\psi_{0}, k\right), k\right)\right)-\Gamma t,
\end{aligned}
$$

for $M$ and $k$ as in the previous case.

* Rotations: $\delta p_{r}<0<\bar{h}$ or $0<\delta p_{r}<\bar{h}$, and $x_{1}<0<1<x_{2}$. Then

$$
\begin{aligned}
x(t) & =\frac{x_{1} \operatorname{sn}^{2}\left(M t+\psi_{0}, k\right)}{\operatorname{sn}^{2}\left(M t+\psi_{0}, k\right)-1+x_{1}}, \\
r(t)-r(0) & =-\frac{\delta x_{1}}{M}\left(\Pi\left(\frac{1}{1-x_{1}}, \operatorname{am}\left(M t+\psi_{0}, k\right), k\right)-\Pi\left(\frac{1}{1-x_{1}}, \operatorname{am}\left(\psi_{0}, k\right), k\right)\right)+\left(-\gamma_{+} t+\delta x_{1}\right) t,
\end{aligned}
$$

for

$$
M^{2}=\delta^{2} x_{2}\left(1-x_{1}\right), \quad k^{2}=\frac{x_{2}-x_{1}}{x_{2}\left(1-x_{1}\right)}
$$

* Separatrices. There are two classes of separatrices that divide short and long periodic orbits, and long periodic orbits and rotations respectively. They are characterized by $x_{1}=0$ or $x_{2}=1$. The explicit parametrization can be obtained as a limit case of the above formulas as $k \rightarrow 1$.

IIb. $\left|p_{r}\right| \geq|\delta| / 2$ (see Fig. 3, left and right). For this values of parameters there are no small periodic orbits. More precisely, they are:

* Parallel solutions: as before, on the level sets $\bar{h}=\delta p_{r}$ and $\bar{h}=0$ we have parallel solutions $\varphi=0$ and $\varphi=\pi / 2$, which in in the meridian case results horizontal and vertical axes on the $y z$-plane;

* There are two families of long orbits: centered at $\pi / 2$ (if $p_{r} / \delta<-1 / 2$ ) and at 0 (if $\left.p_{r} / \delta<1 / 2\right)$, existing on the level sets $\bar{h}<\delta p_{r}<0$ and $0<\bar{h}<\delta p_{r}$. Their explicit parametrization is the same as for the two classes of long orbits described in IIa;

$*$ Rotational motions live on the level sets $\bar{h}>\delta p_{r}>0$ and $\bar{h}<\delta p_{r}<0$. They can be parametrized as rotations in case IIa.

IIc. $p_{r}=0$ (see Fig. 3, center). In this case, there are no long periodic orbits, and all quadrants of the $y z$-plane are symmetric. In each quadrant there are radial solutions corresponding to the equilibrium points $\varphi=\pi / 4 \bmod \pi / 2$ lying on the level set $\bar{h}=-\delta^{2} / 8$, which give rise to families of short periodic orbits. In addition, there are equilibrium points of hyperbolic type $\varphi=0 \bmod \pi / 2$ on the level set $\bar{h}=0$. The level sets $\bar{h}>0$ carry on rotations. The parameterization of these trajectories is analogous to the case IIa.

\section{REFERENCES}

1. M. Abramowitz and I. A. Stegun, Handbook of Mathematical Functions, Dover Publications, New York (1964).

2. A. Agrachev, U. Boscain, and M. Sigalotti, "A Gauss-Bonnet-like formula on two-dimensional almost Riemannian manifolds," Discr. Contin. Dynam. Syst. A, 20, 801-822 (2008).

3. B. Bonnard and M. Chyba, "Singular trajectories and their role in control theory," in: Math. Appl., 40, Springer-Verlag, Berlin (2003).

4. B. Bonnard and J.-B. Caillau, Singular Metrics on the Two-Sphere in Space Mechanics, HAL (2008). 
5. B. Bonnard, O. Cots, N. Shcherbakova, and D. Sugny, "The energy minimization problem for two-level dissipative quantum systems," J. Math. Phys., 51 (2010), DOI: 10.1063/1.3479390.

6. B. Bonnard, J.-B. Caillau, and O. Cots, "Energy minimization in two-level dissipative quantum control: the integrable case," in: Proc. 8th AIMS Conf. on Dynamical Systems, Differential Equations and Applications, Dresden (2011), pp. 198-208.

7. U. Boscain and P. Mason, "Time minimal trajectories for a spin $1 / 2$ particle in a magnetic field," J. Math. Phys., 47, No. 6 (2006).

8. J. B. Caillau, O. Cots, and J. Gergaud, Hampath, apo.enseeiht.fr/hampath.

9. M. P. do Carmo, Riemannian Geometry, Birkhäuser, Boston (1992).

10. H. T. Davies, Introduction to Nonlinear Differential and Integral Equations, Dover Publications, New York (1962).

11. D. F. Lawden, Elliptic Functions and Applications, Springer-Verlag, New York (1989).

12. V. V. Nemytskii and V. V. Stepanov, Qualitative Theory of Differential Equations, Princeton Univ. Press (1960).

B. Bonnard

Institut de Mathématiques de Bourgogne and ENSEEIHT-IRIT INP Toulouse, France O. Cots

Institut de Mathématiques de Bourgogne and ENSEEIHT-IRIT INP Toulouse, France N. Shcherbakova

Institut de Mathématiques de Bourgogne and ENSEEIHT-IRIT INP Toulouse, France

E-mail: nshcherb@enseeiht.fr 